NISSUNA UMANA INVESTIGAZIONE SI PUO DIMANDARE VERA SCIENZIA S'ESSA NON PASSA PER LE MATEMATICHE DIMOSTRAZIONI LEONARDO DA VINCI

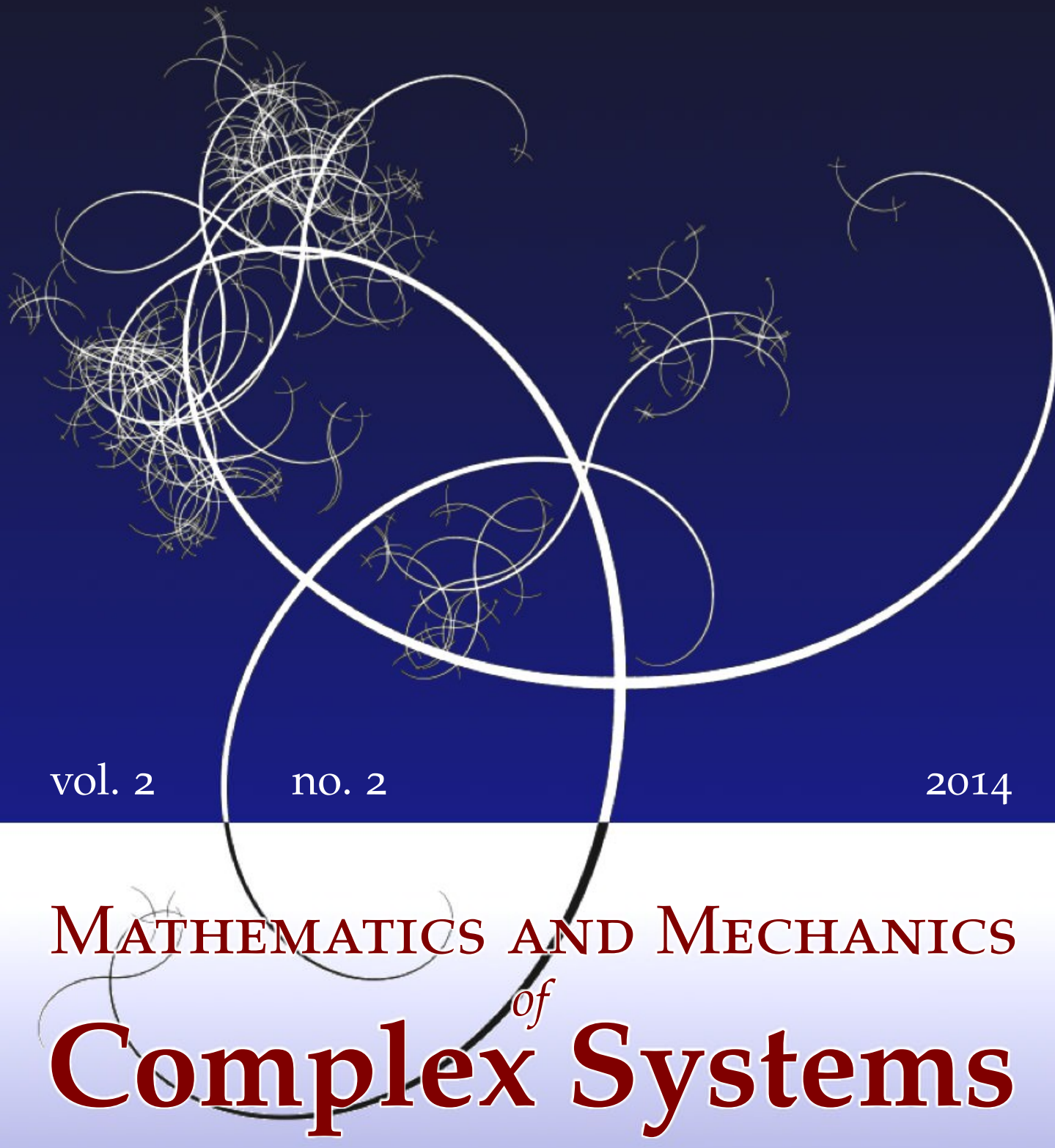

Thi Bach Tuyet Dang, Laurence Halpern and Jean-Jacques Marigo ASYMPTOTIC ANALYSIS OF SMALL DEFECTS NEAR A SINGULAR POINT IN ANTIPLANE ELASTICITY, WITH AN APPLICATION TO THE NUCLEATION OF A CRACK AT A NOTCH 


\title{
ASYMPTOTIC ANALYSIS OF SMALL DEFECTS NEAR A SINGULAR POINT IN ANTIPLANE ELASTICITY, WITH AN APPLICATION TO THE NUCLEATION OF A CRACK AT A NOTCH
}

\author{
Thi Bach Tuyet Dang, Laurence Halpern and JeAn-Jacques Marigo
}

\begin{abstract}
We use matching asymptotic expansions to treat the antiplane elastic problem associated with a small defect located at the tip of a notch. In a first part, we develop the asymptotic method for any type of defect and present the sequential procedure which allows us to calculate the different terms of the inner and outer expansions at any order. This requires in particular separating in each term its singular part from its regular part. In a second part, the asymptotic method is applied to the case of a crack of variable length located at the tip of a given notch. We show that the first two nontrivial terms of the expansion of the energy release rate are sufficient to well approximate the dependence of the energy release rate on the crack length in the range of values of the length which are sufficient to treat the problem of nucleation. This problem is considered in the last part where we compare the nucleation and the propagation of a crack predicted by two different models: the classical Griffith law and the Francfort-Marigo law based on an energy minimization principle. Several numerical results illustrate the interest of the method.
\end{abstract}

\section{Introduction}

A major issue in fracture mechanics is how to model the initiation of a crack in a sound material; see [Bourdin et al. 2008]. There are two difficulties: the first one is to propose a law able to predict that nucleation; the second is a purely numerical issue. Indeed, it is difficult to compute with good accuracy the energy release rate associated with a crack of small length which appears at the tip of a notch; see [Marigo 2010]. The classical finite element method (FEM) leads to inaccurate

\section{Communicated by Francesco dell'Isola.}

This work was partially supported by the French Agence Nationale de la Recherche (ANR), under grant epsilon (BLAN08-2_312370) "Domain decomposition and multi-scale computations of singularities in mechanical structures".

MSC2000: 35A15, 35B40, 35C20, 74A45, 74G70, 74R10.

Keywords: brittle fracture, variational methods, asymptotic methods, singularities. 
results because of the overlap of two singularities which cannot be correctly captured by this method: one is due to the tip of the notch; the other is due to the tip of the crack. A specific method of approximation based on asymptotic expansions is preferable as it is developed in analogous situations with localized defects; see for instance [Abdelmoula and Marigo 2000; Abdelmoula et al. 2010; Bilteryst and Marigo 2003; Bonnaillie-Noël et al. 2010; 2011; David et al. 2012; Geymonat et al. 2011; Leguillon 1989; Marigo and Pideri 2011; Vidrascu et al. 2012]. The first part of the present paper is devoted to the presentation of this matched asymptotic method (shortly, the MAM) in the case of a defect (which includes the case of a crack) located at the tip of a notch in the simplified context of antiplane linear elasticity. Therefore, our approach can be considered as a particular case of the previous works which have been devoted to the study of elliptic problems in corner domains, like [Dauge 1988; Dauge et al. 2010; Grisvard 1985; 1986]. However, a major difference is that we want to use these asymptotic methods to predict the nucleation or the propagation of defects (like cracks) near those singular points. The second and third parts of our paper will be devoted to this task. This requires, of course, to introduce a criterion of nucleation. This delicate issue has not received a definitive answer at the present time and it was considered for a long time as a problem which could not be solved in the framework of Griffith's theory of fracture [Bui 1978; Cherepanov 1979; Lawn 1993; Leblond 2003]. The main invoked reason is that the release of energy due to a small crack tends to zero when the length of the crack tends to zero; see [Chambolle et al. 2008; Marigo 2010]. Therefore, according to the Griffith criterion which states that the crack can propagate only when the energy release rate reaches a critical value characteristic of the material, no nucleation is possible because the energy release rate vanishes when there is no preexisting crack. This limitation of Griffith's theory was one of the motivations which led Francfort and Marigo [1998] to replace the Griffith criterion by a principle of least energy, in the spirit of the original idea of [Griffith 1921]. It turns out that the principle of least energy is really able to predict the nucleation of cracks in a sound body. However, as it was generically proved in [Chambolle et al. 2008; Francfort and Marigo 1998], the nucleation is necessarily brutal in the sense that a crack of finite length suddenly appears at a critical loading. Accordingly, we propose to revisit the problem of nucleation of a crack at the tip of a notch by comparing the two criteria. One of our goals is to use the MAM to obtain semianalytical expressions for the critical loading at which a crack appears and the length of the nucleated crack.

Specifically, the paper is organized as follows. Section 2 is devoted to the description of the MAM on a generic antiplane linear elastic problem where the body contains a defect near the tip of a notch. We first decompose the solution into two expansions: the outer expansion is valid far enough from the tip of the notch while the inner expansion is valid in a neighborhood of the tip of the notch. These 
expansions contain a sequence of inner and outer terms which are solutions of inner and outer problems and are connected by the matching conditions. Moreover each term contains a regular and a singular part. We explain how all the terms and the coefficients entering in their singular and regular parts are sequentially determined. The section finishes with an example where the exact solution is obtained in closed form and hence where we can verify the relevance of the MAM.

In Section 3, the MAM is applied to the case where the defect is a crack. Its main goal is to compute with good accuracy the energy release rate associated with a crack of small length near the tip of the notch. Indeed, it is a real issue in the case of a genuine notch (as opposed to a crack) because the energy release rate starts from 0 when the length of the nucleated crack is 0 , then is rapidly increasing with the length of the crack before reaching a maximum and is finally decreasing. Accordingly, after the setting of the problem, the computation of the energy release rate by the FEM is described, and the reason why the numerical results are less accurate when the crack length is small is given. Then, the MAM is used to compute the energy release rate for small values of the crack length. As expected, the computation shows that, the smaller the size of the defect, the more accurate is the approximation by the MAM at a certain order. It even appears that very accurate results can be obtained by computing a small number of terms in the matched asymptotic expansions. We discuss also the influence of the angle of the notch on the accuracy of the results, this angle playing an important role in the process of nucleation (because, in particular, the length $l_{\mathrm{m}}$ at which the maximum of the energy release rate is reached depends on the angle of the notch). It turns out that when the notch is sufficiently sharp, i.e., sufficiently close to a crack, the first two nontrivial terms of the expansion of the energy release rate are sufficient to capture with very good accuracy the dependence of the energy release rate on the crack length.

In Section 4, we study the problem of crack nucleation at the tip of a notch. We first introduce the two competing evolution laws, i.e., the G-law and the FM-law: the first one is the usual Griffith's law based on the criterion of critical energy release rate; the second is that introduced in [Francfort and Marigo 1998], which is based on the concept of energy minimization. We recall some general results previously established in [Marigo 2010] and extend them to the present case of a notch-shaped body in an antiplane setting. By virtue of the good approximation given by the MAM, we are able to solve the evolution problem in a quasiclosed form, the solution depending only on two coefficients that must be computed by the FEM. This permits a qualitative and quantitative comparison of the two laws. 


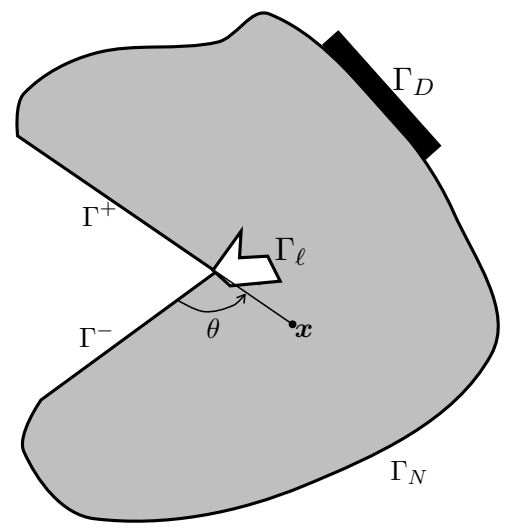

Figure 1. The domain $\Omega_{l}$ for the real problem.
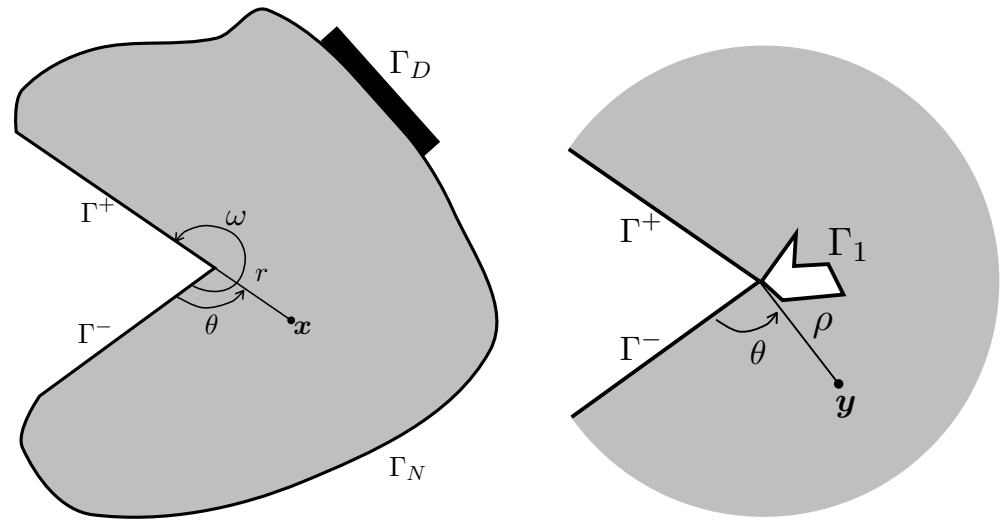

Figure 2. The domains $\Omega_{0}$ and $\Omega^{\infty}$ for, respectively, the outer (left) and the inner (right) problems.

\section{The real problem and its expansion by the matched asymptotic method}

2.1. The real problem. Here, we consider a small geometrical defect of size $l$ (like a crack or a void) located near the corner of a notch; see Figure 1. The geometry of the notch is characterized by its angle $\omega$; see Figure 2. The tip of the notch is taken as the origin of the space. We will introduce two scales of coordinates: the "macroscopic" coordinates $\boldsymbol{x}=\left(x_{1}, x_{2}\right)$ used in the outer domain, and the "microscopic" coordinates $\boldsymbol{y}=\boldsymbol{x} / l=\left(y_{1}, y_{2}\right)$ used in the neighborhood of the tip of the notch where the defect is located; see Figure 2. In the case of a crack, the axis $x_{1}$ is chosen in such a way that the crack corresponds to the line segment $(0, l) \times\{0\}$. The unit vector orthogonal to the $\left(x_{1}, x_{2}\right)$ plane is denoted by $\boldsymbol{e}_{3}$. 
The natural reference configuration of the sound two-dimensional body is $\Omega_{0}$, while the associated body which contains a defect of size $l$ is $\Omega_{l}$. The part of the boundary of $\Omega_{l}$ which is due to the defect is denoted by $\Gamma_{l}$; i.e.,

$$
\Gamma_{l}=\partial \Omega_{l} \backslash \partial \Omega_{0},
$$

and $\Gamma_{l}$ is contained in the disk of center $(0,0)$ and radius $l$. In the case of a crack, $\Gamma_{l}$ is the crack itself; i.e., $\Gamma_{l}=(0, l) \times\{0\}$. The two edges of the notch are denoted by $\Gamma^{+}$and $\Gamma^{-}$. To simplify the presentation, it is assumed that they are not modified by the introduction of the defect; see Figure 1 . When using polar coordinates $(r, \theta)$, the pole is the tip of the notch and the origin of the polar angle is the edge $\Gamma^{-}$. Accordingly, we have

$$
r=|x|, \quad \Gamma^{-}=\left\{(r, \theta): 0<r<r^{*}, \theta=0\right\}, \quad \Gamma^{+}=\left\{(r, \theta): 0<r<r^{*}, \theta=\omega\right\} .
$$

This body is made of an elastic isotropic material whose shear modulus is $\mu>0$. It is submitted to a loading such that the displacement field at equilibrium $\boldsymbol{u}_{l}$ be antiplane; i.e.,

$$
\boldsymbol{u}_{l}(\boldsymbol{x})=u_{l}\left(x_{1}, x_{2}\right) \boldsymbol{e}_{3},
$$

where the subscript letter $l$ is used as a reminder that the real displacement depends on the size of the defect. We assume that the body forces are zero and then $u_{l}$ must be an harmonic function in order to satisfy the equilibrium equations in the bulk:

$$
\Delta u_{l}=0 \quad \text { in } \Omega_{l} .
$$

The edges of the notch are free while $\Gamma_{l}$ is submitted to a density of (antiplane) surface forces. Accordingly, the boundary conditions on $\Gamma_{l}$ and $\Gamma^{ \pm}$are

$$
\frac{\partial u_{l}}{\partial v}=0 \quad \text { on } \Gamma^{ \pm}, \quad \frac{\partial u_{l}}{\partial v}(\boldsymbol{x})=\frac{g(\boldsymbol{y})}{l} \quad \text { on } \Gamma_{l} .
$$

In (4), $v$ denotes the unit outer normal vector to $\Omega_{l}$, and we assume that the density of (antiplane) surface forces depends on the microscopic variable $\boldsymbol{y}$ and has a magnitude of the order of $1 / l$.

The remaining part of the boundary of $\Omega_{l}$ is divided into two parts: $\Gamma_{D}$ where the displacement is prescribed and $\Gamma_{N}$ where (antiplane) surface forces are prescribed. Specifically, we have

$$
u_{l}=f \quad \text { on } \Gamma_{D}, \quad \frac{\partial u_{l}}{\partial v}=h \quad \text { on } \Gamma_{N} .
$$

The following proposition is a characterization of those functions which are harmonic in an angular sector and whose normal derivatives vanish on the edges of the sector. It is of constant use throughout the paper. 
Proposition 1. Let $r_{1}$ and $r_{2}$ be such that $0 \leq r_{1}<r_{2} \leq+\infty$ and let $\mathscr{D}_{r_{1}}^{r_{2}}$ be the angular sector

$$
\mathscr{D}_{r_{1}}^{r_{2}}=\left\{(r, \theta): r \in\left(r_{1}, r_{2}\right), \theta \in(0, \omega)\right\} .
$$

Then any function $u$ which is harmonic in $\mathscr{D}_{r_{1}}^{r_{2}}$ and which satisfies the Neumann condition $\partial u / \partial \theta=0$ on the sides $\theta=0$ and $\theta=\omega$ can be expanded as

$$
u(r, \theta)=\mathrm{a}_{0} \ln (r)+\mathrm{d}_{0}+\sum_{n \in \mathbb{N}^{*}}\left(\mathrm{a}_{n} r^{-n \lambda}+\mathrm{d}_{n} r^{n \lambda}\right) \cos (n \lambda \theta), \quad \lambda=\frac{\pi}{\omega},
$$

where the $\mathrm{a}_{n}$ and the $\mathrm{d}_{n}$ constitute two sequences of real numbers which are characteristic of $u$.

Proof. Since the normal derivative vanishes at $\theta=0$ and $\theta=\omega, u(r, \theta)$ can be expanded in a Fourier series as

$$
u(r, \theta)=\sum_{n \in \mathbb{N}} f_{n}(r) \cos (n \lambda \theta) .
$$

In order that $u$ be harmonic, the functions $f_{n}$ must satisfy $r^{2} f_{n}^{\prime \prime}+r f_{n}^{\prime}-n^{2} \lambda^{2} f_{n}=0$ for each $n$. We easily deduce that $f_{0}(r)=\mathrm{a}_{0} \ln (r)+\mathrm{d}_{0}$ and $f_{n}(r)=\mathrm{a}_{n} r^{-n \lambda}+\mathrm{d}_{n} r^{n \lambda}$ for $n \geq 1$.

2.2. The matching asymptotic method (MAM). We will write two asymptotic expansions of $u_{l}$ in terms of the small parameter $l$. The inner expansion is valid in the neighborhood of the tip of the notch, while the outer expansion is valid far from this tip. These two expansions will be matched in an intermediate zone.

2.2.1. The outer expansion. Far from the tip of the notch, i.e., for $r \gg l, u_{l}$ does not see the notch, and we assume that it can be expanded as

$$
u_{l}(\boldsymbol{x})=\sum_{i \in \mathbb{N}} l^{i \lambda} u^{i}(\boldsymbol{x})
$$

In (7), even if this expansion is valid far enough from $r=0$ only, $u^{i}$ must be defined in the whole outer domain $\Omega_{0}$ which corresponds to the sound body; see Figure 2 (left). Inserting this expansion into (3), (4), and (5) yields the sequence of problems for the $u^{i}$ :

The first outer problem, $i=0$ :

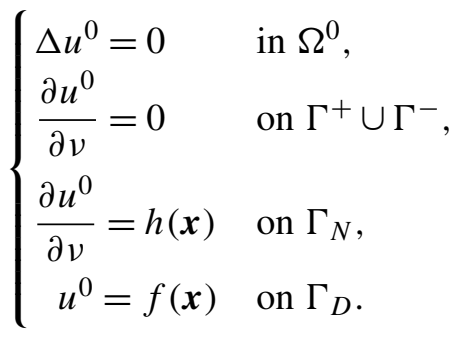


The other outer problems, $i \geq 1$ :

$$
\begin{cases}\Delta u^{i}=0 & \text { in } \Omega^{0}, \\ \frac{\partial u^{i}}{\partial v}=0 & \text { on } \Gamma^{+} \cup \Gamma^{-}, \\ \frac{\partial u^{i}}{\partial v}=0 & \text { on } \Gamma_{N}, \\ u^{i}=0 & \text { on } \Gamma_{D} .\end{cases}
$$

Moreover, the behavior of $u^{i}$ in the neighborhood of $r=0$ is singular and the singularity will be given by the matching conditions.

2.2.2. The inner expansion. Near the tip of the notch, i.e., for $r \ll 1$, we assume that the displacement field $u_{l}$ can be expanded as

$$
u_{l}(\boldsymbol{x})=\ln (l) \sum_{i \in \mathbb{N}} l^{i \lambda} w^{i}(\boldsymbol{y})+\sum_{i \in \mathbb{N}} l^{i \lambda} v^{i}(\boldsymbol{y}), \quad \boldsymbol{y}=\frac{\boldsymbol{x}}{l} .
$$

In (10), even if this expansion is valid only in the neighborhood of $r=0$, the fields $v^{i}$ and $w^{i}$ must be defined in the infinite inner domain $\Omega^{\infty}$. The domain $\Omega^{\infty}$ is the infinite angular sector $\mathscr{D}_{0}^{\infty}$ of the $\left(y_{1}, y_{2}\right)$ plane, from which the rescaled defect of size 1 is removed; see Figure 2 (right). Accordingly, the rescaled boundary $\Gamma_{1}$ of the defect is

$$
\Gamma_{1}=\partial \Omega^{\infty} \backslash \partial \mathscr{D}_{0}^{\infty} .
$$

(In the case of a crack, $\Gamma_{1}=(0,1) \times\{0\}$.) Inserting this expansion into the set of equations constituting the real problem yields the sequence of problems for the $v^{i}$ :

The first inner problem, $i=0$ :

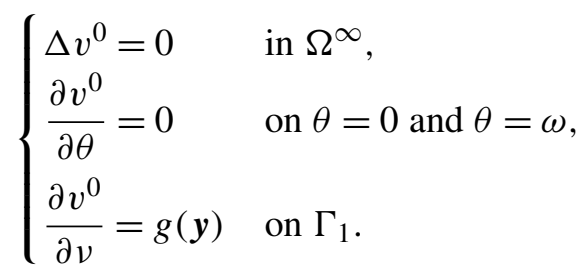

The other inner problems, $i \geq 1$ :

$$
\begin{cases}\Delta v^{i}=0 & \text { in } \Omega^{\infty}, \\ \frac{\partial v^{i}}{\partial \theta}=0 & \text { on } \theta=0 \text { and } \theta=\omega, \\ \frac{\partial v^{i}}{\partial v}=0 & \text { on } \Gamma_{1} .\end{cases}
$$


The $w^{i}$ must satisfy, for every $i \geq 0$, the same equations as the $v^{i}$ for $i \geq 1$. To complement the set of equations, the behavior at infinity of the $v^{i}$ and the $w^{i}$ must be included. It is obtained by the matching conditions from the outer problems.

2.2.3. Matching conditions. In any sector $\mathscr{D}_{0}^{r_{2}}$ with $l \ll r_{2} \ll 1$, the displacement fields $u^{i}$ in the outer expansion are harmonic and satisfy homogeneous Neumann boundary conditions on the edges. Therefore Proposition 1 applies, and

$$
u^{i}(\boldsymbol{x})=\mathrm{a}_{0}^{i} \ln (r)+\mathrm{d}_{0}^{i}+\sum_{n \in \mathbb{N}^{*}}\left(\mathrm{a}_{n}^{i} r^{-n \lambda}+\mathrm{d}_{n}^{i} r^{n \lambda}\right) \cos (n \lambda \theta) .
$$

As for the inner expansion, the displacement fields $v^{i}$ and $w^{i}$ are harmonic in the sector $\mathscr{D}_{1}^{\infty}$ of the $\boldsymbol{y}$ plane and satisfy homogeneous Neumann boundary conditions on the edges. Therefore Proposition 1 applies, with the microscopic coordinates $y$ and $\rho=|\boldsymbol{y}|=r / l$ replacing the macroscopic coordinates $\boldsymbol{x}$ and $r$ :

$$
\begin{aligned}
& v^{i}(\boldsymbol{y})=\mathrm{c}_{0}^{i} \ln (\rho)+\mathrm{b}_{0}^{i}+\sum_{n \in \mathbb{N}^{*}}\left(\mathrm{c}_{n}^{i} \rho^{-n \lambda}+\mathrm{b}_{n}^{i} \rho^{n \lambda}\right) \cos (n \lambda \theta), \\
& w^{i}(\boldsymbol{y})=\mathrm{e}_{0}^{i} \ln (\rho)+\mathrm{f}_{0}^{i}+\sum_{n \in \mathbb{N}^{*}}\left(\mathrm{e}_{n}^{i} \rho^{-n \lambda}+\mathrm{f}_{n}^{i} \rho^{n \lambda}\right) \cos (n \lambda \theta) .
\end{aligned}
$$

The outer expansion and the inner expansion are both valid in any intermediate zone $\mathscr{D}_{r_{1}}^{r_{2}}$ such that $l \ll r_{1}<r_{2} \ll 1$. Inserting (14) into the outer expansion (7) with $r=l \rho$ leads to

$$
\begin{aligned}
u_{l}(\boldsymbol{x})= & \sum_{i \in \mathbb{N}} \ln (l) l^{i \lambda} \mathrm{a}_{0}^{i} \\
& +\sum_{i \in \mathbb{N}} l^{i \lambda}\left(\mathrm{a}_{0}^{i} \ln (\rho)+\mathrm{d}_{0}^{i}+\sum_{n \in \mathbb{N}^{*}}\left(\mathrm{a}_{n}^{i+n} \rho^{-n \lambda}+\mathrm{d}_{n}^{i-n} \rho^{n \lambda}\right) \cos (n \lambda \theta)\right),
\end{aligned}
$$

with the convention that $\mathrm{d}_{n}^{i-n}=0$ when $n>i$. Inserting (15) and (16) into the inner expansion (10) leads to

$$
\begin{aligned}
u_{l}(\boldsymbol{x})=\sum_{i \in \mathbb{N}} \ln (l) l^{i \lambda}\left(\mathrm{e}_{0}^{i} \ln (\rho)+\mathrm{f}_{0}^{i}+\sum_{n \in \mathbb{N}^{*}}\left(\mathrm{e}_{n}^{i} \rho^{-n \lambda}+\mathrm{f}_{n}^{i} \rho^{n \lambda}\right) \cos (n \lambda \theta)\right) \\
+\sum_{i \in \mathbb{N}} l^{i \lambda}\left(\mathrm{c}_{0}^{i} \ln (\rho)+\mathrm{b}_{0}^{i}+\sum_{n \in \mathbb{N}^{*}}\left(\mathrm{c}_{n}^{i} \rho^{-n \lambda}+\mathrm{b}_{n}^{i} \rho^{n \lambda}\right) \cos (n \lambda \theta)\right) .
\end{aligned}
$$

Both expansions (17) and (18) are valid provided that $1 \ll \rho \ll 1 / l$. Identification of these expansions provides the connections between the coefficients of the inner and outer expansions described in Table 1.

Remark 1. From Table 1 can be deduced that the fields $w^{i}$ are constant in the whole inner domain:

$$
w^{i}(\boldsymbol{y})=\mathrm{a}_{0}^{i} \quad \text { for all } \boldsymbol{y} \in \Omega^{\infty} \text { and all } i \geq 0 .
$$




$$
\begin{array}{|lc}
\mathrm{e}_{n}^{i}=0 & i \geq 0, n \geq 0 \\
\mathrm{f}_{0}^{i}=\mathrm{a}_{0}^{i} & i \geq 0 \\
\mathrm{f}_{n}^{i}=0 & i \geq 0, n \geq 1 \\
\mathrm{a}_{n}^{i}=0 & n>i \geq 0 \\
\mathrm{c}_{n}^{i}=\mathrm{a}_{n}^{i+n} & i \geq 0, n \geq 0 \\
\mathrm{~b}_{n}^{i}=0 & n>i \geq 0 \\
\mathrm{~d}_{n}^{i}=\mathrm{b}_{n}^{i+n} & i \geq 0, n \geq 0
\end{array}
$$

Table 1. The relations between the coefficients of the inner and outer expansions given by the matching conditions.

Therefore, these fields will be determined once the constants $a_{0}^{i}$ are known.

2.2.4. The singular behavior of the $u^{i}$ and the $v^{i}$. From the matching conditions can be read the behavior of $u^{i}$ in the neighborhood of $r=0$ and the behavior of $v^{i}$ at infinity. In particular, the form of their singularities is visible, according to the following definition.

Definition 1. A field $u$ defined in $\Omega_{0}$ is regular in $\Omega_{0}$ if $u \in H^{1}\left(\Omega_{0}\right)$; i.e., $u \in$ $L^{2}\left(\Omega_{0}\right)$ and $\nabla u \in L^{2}\left(\Omega_{0}\right)^{2}$. It is singular otherwise.

A field $u$ defined in the unbounded domain $\Omega^{\infty}$ is regular in $\Omega^{\infty}$ if $\nabla u \in$ $\left(L^{2}\left(\Omega^{\infty}\right)\right)^{2}$ and $\lim _{\rho \rightarrow \infty} u(\rho, \theta)=0$. It is singular otherwise.

Remark 2. In other words, a field is regular if the associated elastic energy is finite. It is singular otherwise. In the case of the unbounded domain $\Omega^{\infty}$, a constant field has finite energy, but the condition at infinity is added in order to fix the constant and obtain the uniqueness in the forthcoming boundary value problems.

According to the analysis in the previous subsection, the field $u^{0}$ can be expanded in a neighborhood of the tip of the notch as

$$
u^{0}(\boldsymbol{x})=\mathrm{a}_{0}^{0} \ln (r)+\sum_{n \in \mathbb{N}} \mathrm{b}_{n}^{n} r^{n \lambda} \cos (n \lambda \theta)
$$

In the domain $\Omega_{0}, \ln (r)$ is singular, whereas $r^{n \lambda} \cos (n \lambda \theta)$ is regular for $n \geq 0$, in the sense of Definition 1. Accordingly, $u^{0}$ is split into its singular and regular parts as follows:

$$
\begin{gathered}
u^{0}(\boldsymbol{x})=u_{S}^{0}(\boldsymbol{x})+\bar{u}^{0}(\boldsymbol{x}), \\
u_{S}^{0}(\boldsymbol{x})=\mathrm{a}_{0}^{0} \ln (r), \quad \bar{u}^{0} \in H^{1}\left(\Omega_{0}\right) .
\end{gathered}
$$


In the same way, for $i \geq 1$, the field $u^{i}$ can be expanded in a neighborhood of the tip of the notch as

$$
u^{i}(\boldsymbol{x})=\mathrm{a}_{0}^{i} \ln (r)+\sum_{n=1}^{i} \mathrm{a}_{n}^{i} r^{-n \lambda} \cos (n \lambda \theta)+\sum_{n \in \mathbb{N}} \mathrm{b}_{n}^{i+n} r^{n \lambda} \cos (n \lambda \theta)
$$

Since $r^{-n \lambda} \cos (n \lambda \theta)$ is singular (for $n \geq 0$ ) in the sense of Definition $1, u^{i}$ is split into its singular and regular parts as follows:

$$
\begin{gathered}
u^{i}(\boldsymbol{x})=u_{S}^{i}(\boldsymbol{x})+\bar{u}^{i}(\boldsymbol{x}), \\
u_{S}^{i}(\boldsymbol{x})=\mathrm{a}_{0}^{i} \ln (r)+\sum_{n=1}^{i} \mathrm{a}_{n}^{i} r^{-n \lambda} \cos (n \lambda \theta), \quad \bar{u}^{i} \in H^{1}\left(\Omega_{0}\right) .
\end{gathered}
$$

For the fields $v^{i}$ of the inner expansion, the behavior at infinity comes into play. By virtue of the analysis in the previous subsection, the field $v^{i}$ for $i \geq 0$ can be expanded for large $\rho$ as

$$
v^{i}(\boldsymbol{y})=\mathrm{a}_{0}^{i} \ln (\rho)+\sum_{n=0}^{i} \mathrm{~b}_{n}^{i} \rho^{n \lambda} \cos (n \lambda \theta)+\sum_{n \in \mathbb{N}^{*}} \mathrm{a}_{n}^{i+n} \rho^{-n \lambda} \cos (n \lambda \theta) .
$$

The field $\ln (\rho)$ as well as the fields $\rho^{n \lambda} \cos (n \lambda \theta)$, for $n \geq 0$, are singular in $\Omega^{\infty}$ in the sense of Definition 1 (even the constant field 1 corresponding to $n=0$ is singular). Since the fields $\rho^{-n \lambda} \cos (n \lambda \theta)$ are regular when $n \geq 1, v^{i}$ is split into its singular and regular parts as follows:

$$
\begin{gathered}
v^{i}(\boldsymbol{y})=v_{S}^{i}(\boldsymbol{y})+\bar{v}^{i}(\boldsymbol{y}), \\
v_{S}^{i}(\boldsymbol{y})=\mathrm{a}_{0}^{i} \ln (\rho)+\sum_{n=0}^{i} \mathrm{~b}_{n}^{i} \rho^{n \lambda} \cos (n \lambda \theta), \quad \nabla \bar{v}^{i} \in L^{2}\left(\Omega^{\infty}\right), \quad \lim _{|\boldsymbol{y}| \rightarrow \infty} \bar{v}^{i}(\boldsymbol{y})=0 .
\end{gathered}
$$

Remark 3. This analysis of the singularities shows that the singular parts of the fields $u^{i}$ and $v^{i}$ will be known once the coefficients $\mathrm{a}_{n}^{i}$ and $\mathrm{b}_{n}^{i}$ are determined for $0 \leq n \leq i$.

2.2.5. The problems defining the regular parts $\bar{u}^{i}$ and $\bar{v}^{i}$. The singular parts $\left(u_{S}^{i}, v_{S}^{i}\right)$ are harmonic and satisfy the homogeneous Neumann boundary conditions on the edges of the notch. Therefore the regular parts are harmonic too, with data expressed in terms of the singular fields. 
The first outer problem, $i=0$ : Find $\bar{u}^{0}$ regular in $\Omega_{0}$ such that

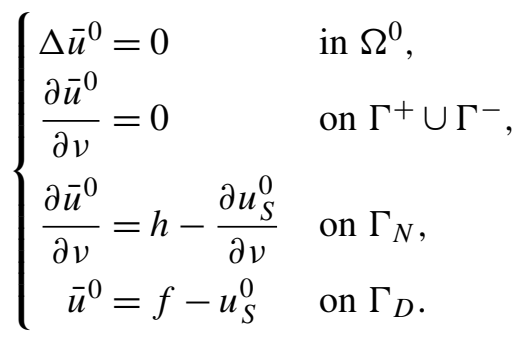

The other outer problems, $i \geq 1$ : Find $\bar{u}^{i}$ regular in $\Omega_{0}$ such that

$$
\left\{\begin{aligned}
\Delta \bar{u}^{i} & =0 & & \text { in } \Omega^{0}, \\
\frac{\partial \bar{u}^{i}}{\partial v} & =0 & & \text { on } \Gamma^{+} \cup \Gamma^{-}, \\
\frac{\partial \bar{u}^{i}}{\partial v} & =-\frac{\partial u_{S}^{i}}{\partial v} & & \text { on } \Gamma_{N}, \\
\bar{u}^{i} & =-u_{S}^{i} & & \text { on } \Gamma_{D} .
\end{aligned}\right.
$$

The first inner problem, $i=0$ : Find $\bar{v}^{0}$ regular in $\Omega^{\infty}$ such that

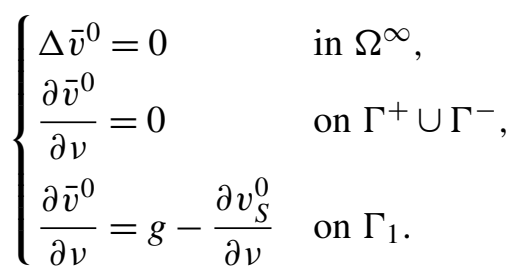

The other inner problems, $i \geq 1$ : Find $\bar{v}^{i}$ regular in $\Omega^{\infty}$ such that

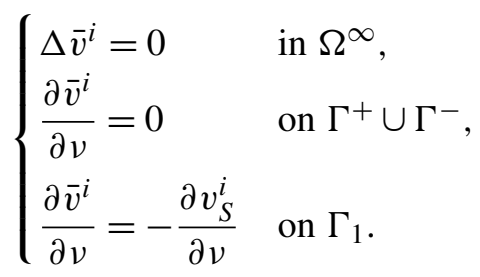

Consider first the outer problems. The well-posedness is a direct consequence of classical results for the Laplace equation:

Proposition 2. Let $i \geq 0$. For a given singular part $u_{S}^{i}$, i.e., if the coefficients $a_{n}^{i}$ are known for all $n$ such that $0 \leq n \leq i$, then there exists a unique solution $\bar{u}^{i}$ of (30) (or of (29) when $i=0)$. Consequently, the coefficients $\mathrm{b}_{n}^{i+n}$ are then determined for all $n \geq 0$. 
As for the inner problems, since they are Neumann problems (except for the condition at infinity), defined in an infinite domain, more care must be taken. The well-posedness is ensured by a compatibility condition, as stated in Proposition 3.

Proposition 3. Let $i \geq 0$. For given $\mathrm{b}_{n}^{i}$ with $0 \leq n \leq i$, there exists a regular solution $\bar{v}^{i}$ for the $i$-th inner problem if and only if the coefficient $\mathrm{a}_{0}^{i}$ is such that

$$
\mathrm{a}_{0}^{0}=-\frac{1}{\omega} \int_{\Gamma_{1}} g(s) d s, \quad \mathrm{a}_{0}^{i}=0 \quad \text { for } i \geq 1 .
$$

Moreover, if this condition is satisfied, then the solution is unique and therefore the coefficients $\mathrm{a}_{n}^{i+n}$ are determined for all $n \geq 0$.

Proof. The inner problems are pure Neumann problems in which no Dirichlet boundary conditions are imposed on the $v^{i}$ except for the condition at infinity. Consequently, they admit a solution (if and) only if the Neumann data satisfy a global compatibility condition. Let us reestablish that condition. Let $\Omega^{R}$ be the part of $\Omega^{\infty}$ included in the ball of radius $R>1$; i.e., $\Omega^{R}=\Omega^{\infty} \cap\{\boldsymbol{y}:|\boldsymbol{y}|<R\}$. Consider first the case $i=0$. Integrating the equation $\Delta v^{0}=0$ over $\Omega^{R}$ and using the boundary conditions leads to

$$
0=\int_{\partial \Omega^{R}} \frac{\partial v^{0}}{\partial v} d s=\int_{0}^{\omega} \frac{\partial v^{0}}{\partial \rho}(R, \theta) R d \theta+\int_{\Gamma_{1}} g(s) d s .
$$

Using (26) yields

$$
R \frac{\partial v^{0}}{\partial \rho}(R, \theta)=\mathrm{a}_{0}^{0}+\sum_{n \in \mathbb{N}^{*}} n \lambda\left(-\mathrm{c}_{n}^{0} R^{-n \lambda}+\mathrm{b}_{n}^{0} R^{n \lambda}\right) \cos (n \lambda \theta) .
$$

Since $\int_{0}^{\omega} \cos (n \lambda \theta) d \theta=0$ for all $n \geq 1$, after inserting in (34), the desired condition for $\mathrm{a}_{0}^{0}$ appears. For $i \geq 1$, the same process is applied, and the integral over $\Gamma_{1}$ vanishes, yielding the desired condition.

If the compatibility condition (33) is satisfied, then the existence of a regular solution for $\bar{v}^{i}$ is obtained by standard arguments. Note however that, since $\nabla \bar{v}^{i}$ belongs to $L^{2}\left(\Omega^{\infty}\right), \bar{v}^{i}$ tends to a constant at infinity and this constant is fixed to 0 by the additional regularity condition. As far as the uniqueness is concerned, the solution of this pure Neumann problem is unique up to a constant and the constant is fixed by the condition that $\bar{v}^{i}$ vanishes at infinity.

Once $v^{i}$ is determined, the coefficients $\mathrm{a}_{n}^{i+n}$ are obtained by virtue of Proposition 1 and (26).

Remark 4. If the forces applied to the boundary of the defect are equilibrated, i.e., if $\int_{\Gamma_{1}} g(s) d s=0$, then all the coefficients $a_{0}^{i}$ vanish and hence the terms in $\ln (l)$ disappear in the inner expansion. There are no more logarithmic singularities in the $u^{i}$ and the $v^{i}$. 
2.2.6. The construction of the outer and inner expansions. Recall the relationship between the coefficients $\left(\mathrm{a}_{n}^{j}, \mathrm{~b}_{n}^{j}\right)$ and the singular and regular parts of the $u_{j}$ and $v_{j}$ :

$$
\begin{array}{lll}
u^{j}=u_{S}^{j}+\bar{u}^{j}, & u_{S}^{j} \longleftrightarrow\left(\mathrm{a}_{n}^{j}\right)_{n=0}^{j}, & \bar{u}^{j} \longleftrightarrow\left(\mathrm{b}_{n}^{j+n}\right)_{n \geq 0}, \\
v^{j}=v_{S}^{j}+\bar{v}^{j}, & v_{S}^{j} \longleftrightarrow\left(\mathrm{a}_{0}^{j},\left(\mathrm{~b}_{n}^{j}\right)_{n=0}^{j}\right), & \bar{v}^{j} \longleftrightarrow\left(\mathrm{a}_{n}^{j+n}\right)_{n \geq 0} .
\end{array}
$$

All the coefficients $a_{0}^{j}$ vanish, except for $a_{0}^{0}$, which is given by (33).

The scheme of the algorithm is the following. Suppose $i \geq 1$, and $u^{j}$ and $v^{j}$ are known for $1 \leq j \leq i-1$. The order of operations at step $i$ is the following:

(1) $u_{S}^{i}$ is determined by $\left(\bar{v}^{i-n}\right)_{1 \leq n \leq i}$,

(2) $\bar{u}^{i}$ is determined by $u_{S}^{i}$,

(3) $v_{S}^{i}$ is determined by $\left(\bar{u}^{i-n}\right)_{0 \leq n \leq i}$,

(4) $\bar{v}^{i}$ is determined by $v_{S}^{i}$.

Details are given below.

Initialization:

(S1) Define $a_{0}^{0}$ by (33), and hence $u_{S}^{0}$ by (22).

(S2) From $u_{S}^{0}$, define $\bar{u}^{0}$ by (29), and hence $u^{0}=u_{S}^{0}+\bar{u}^{0}$ is determined.

(S3) Define $\mathrm{b}_{n}^{n}$ for $n \geq 0$ from (20) as the coefficients of $\bar{u}^{0}$; see the next subsection for the practical method. Hence, $v_{S}^{0}=\mathrm{a}_{0}^{0}+\mathrm{b}_{0}^{0} \ln (\rho)$ is determined from (28).

(S4) From $v_{S}^{0}, \bar{v}^{0}$ is computed by (31), and hence $v^{0}=v_{S}^{0}+\bar{v}^{0}$ is determined.

(S5) Define $\mathrm{a}_{n}^{n}$ for $n \geq 1$ from (26) as the coefficients of $\bar{v}^{0}$; see the next subsection for the practical method.

For $i \geq 1$, suppose that $u^{j}$ and $v^{j}$ have been determined, together with the coefficients in (35), for $0 \leq j \leq i-1$.

(R1) Since $\mathrm{a}_{0}^{i}=0$, and writing, for $1 \leq n \leq i, \mathrm{a}_{n}^{i}=\mathrm{a}_{n}^{(i-n)+n}, u_{S}^{i}$ is given by (25), where the coefficients are determined by those of the $\bar{v}^{j}$ for $1 \leq j \leq i-1$.

(R2) $\bar{u}^{i}$ is obtained by solving (30).

(R3) The coefficients $\mathrm{b}_{n}^{i+n}$ for $n \geq 0$ are extracted from $\bar{u}^{i}$ in (23) and (24); see the next subsection for the practical method.

(R4) Since $\mathrm{a}_{0}^{i}=0$, and using $\mathrm{b}_{n}^{i}=\mathrm{b}_{n}^{j+n}$ with $j=i-n, v_{S}^{i}$ is determined from (28).

(R5) $\bar{v}^{i}$ is obtained by solving (32).

(R6) $u^{i}$ and $v^{i}$ are obtained by summing the singular and regular parts.

This iterative method is summarized in Table 2. 


\begin{tabular}{|c|ccccc|}
\hline $\mathrm{a}_{n}^{i} / \mathrm{b}_{n}^{i}$ & $i=0$ & $i=1$ & $i=2$ & $i=3$ & $i=4$ \\
\hline$n=0$ & $(33)$ /Outer 0 & 0/Outer 1 & 0/Outer 2 & 0/Outer 3 & 0/Outer 4 \\
$n=1$ & 0 & Inner 0/Outer 0 & Inner 1/Outer 1 & Inner 2/Outer 2 & Inner 3/Outer 3 \\
$n=2$ & 0 & 0 & Inner 0/Outer 0 & Inner 1/ Outer 1 & Inner 2/Outer 2 \\
$n=3$ & 0 & 0 & 0 & Inner 0/Outer 0 & Inner 1/Outer 1 \\
$n=4$ & 0 & 0 & 0 & 0 & Inner 0/Outer 0 \\
\hline
\end{tabular}

Table 2. Summary of the inductive method to obtain the coefficients $\mathrm{a}_{n}^{i}$ and $\mathrm{b}_{n}^{i}$ : in the corresponding cell is indicated the problem which must be solved.

2.2.7. The practical method for determining the coefficients $\mathrm{a}_{n}^{i}$ and $\mathrm{b}_{n}^{i}$ for $0 \leq n \leq i$. Throughout this section, $\mathscr{b}_{r}$ denotes the arc of the circle of radius $r$ starting on $\Gamma^{-}$ and ending on $\Gamma^{+}$:

$$
\mathscr{C}_{r}=\{(r, \theta): 0 \leq \theta \leq \omega\} .
$$

The coefficients $\mathrm{a}_{n}^{i}$ and $\mathrm{b}_{n}^{i}$ can be obtained by path integrals (which are path independent) as asserted in the following proposition.

Proposition 4. Let $i \geq 0$. Assume that $\bar{v}^{i}$ and $\bar{u}^{i}$ are known. Then:

(1) For $n \geq 1, a_{n}^{i+n}$ is given by the following path integral over $\mathfrak{b}_{\rho}$, which is independent of $\rho$ provided that $\rho>1$ :

$$
a_{n}^{i+n}=\frac{2 \rho^{n \lambda}}{\omega} \int_{0}^{\omega} \bar{v}^{i}(\rho, \theta) \cos (n \lambda \theta) d \theta .
$$

(2) For $n \geq 0, b_{n}^{i+n}$ is given by the following path integral over $\mathscr{C}_{r}$, which is independent of $r$ provided that $0<r<r^{*}$ :

$$
\mathrm{b}_{0}^{i}=\frac{1}{\omega} \int_{0}^{\omega} \bar{u}^{i}(r, \theta) d \theta, \quad \mathrm{b}_{n}^{i+n}=\frac{2 r^{-n \lambda}}{\omega} \int_{0}^{\omega} \bar{u}^{i}(r, \theta) \cos (n \lambda \theta) d \theta \quad \text { for } n \geq 1 .
$$

Proof. The proofs are identical for the two families of coefficients and only that concerning $\mathrm{b}_{n}^{i+n}$ will be given. By (23), $\bar{u}^{i}$ is given for $0<r<r^{*}$ by

$$
\bar{u}^{i}(r, \theta)=\sum_{p \in \mathbb{N}} \mathrm{b}_{p}^{i+p} r^{p \lambda} \cos (p \lambda \theta),
$$

which is for fixed $r$ the Fourier series of $\bar{u}^{i}(r, \cdot)$. Formulas (37) follow.

2.3. Verification in the case of a small cavity. This subsection is devoted to the verification of the construction of the matched asymptotic expansion (MAE) presented in the previous subsections on an example where the exact solution is obtained in a closed form and hence can be directly expanded. Specifically, we consider a Laplace problem posed in a domain which consists of an angular sector 


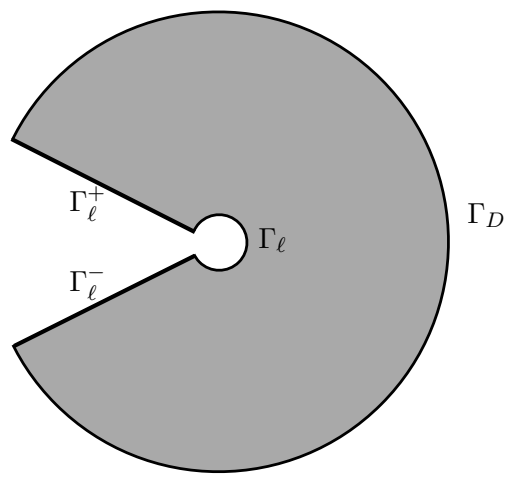

Figure 3. The domain $\Omega_{l}$ in the case of a cavity.

delimited by two arc of circles. The radius of the outer circle is equal to 1 while the radius of the inner circle is $l$; see Figure 3. Thus,

$$
\Omega_{l}=\left\{\boldsymbol{x}=r \cos \theta \boldsymbol{e}_{1}+r \sin \theta \boldsymbol{e}_{2}: r \in(l, 1), \theta \in(0, \omega)\right\} .
$$

The sides of the notch and the inner circle are free and hence the boundary conditions on those parts of the boundary are

$$
\frac{\partial u_{l}}{\partial v}=0 \quad \text { on } \Gamma_{l}^{+} \cup \Gamma_{l}^{-} \cup \Gamma_{l},
$$

where $\Gamma_{l}^{ \pm}=\{(r, \theta): l<r<1, \theta=0$ or $\omega\}, \Gamma_{l}=\{(r, \theta): r=l, 0 \leq \theta \leq \omega\}$. (Note that $\Gamma_{l}^{ \pm}$depend on $l$, contrary to the assumption made in the remaining part of the paper. But that has no influence on the results.) The displacement is prescribed on the outer boundary $\Gamma_{D}$ so that

$$
u_{l}(\boldsymbol{x})=\cos \lambda \theta \quad \text { on } \Gamma_{D}, \quad \lambda=\frac{\pi}{\omega} .
$$

Note that $\Gamma_{N}$ is empty. Assuming that there is no body force, the exact solution of this antiplane elastic problem is given by

$$
u_{l}(\boldsymbol{x})=\left(\frac{l^{2 \lambda}}{1+l^{2 \lambda}} r^{-\lambda}+\frac{1}{1+l^{2 \lambda}} r^{\lambda}\right) \cos \lambda \theta
$$

Inserting the Taylor series of $1 /\left(1+l^{2 \lambda}\right)=\sum_{i \in \mathbb{N}}(-1)^{i}\left(l^{2 \lambda}\right)^{i}$ for $l<1$, the expansion of $u_{l}$ at a given $\boldsymbol{x}$ takes the form

$$
u_{l}(\boldsymbol{x})=r^{\lambda} \cos \lambda \theta+\sum_{n \in \mathbb{N}^{*}} l^{2 n \lambda}\left(r^{-\lambda}-r^{\lambda}\right) \cos \lambda \theta .
$$


Thus (41) corresponds to the outer expansion where the odd terms vanish and the even terms are given by

$$
u^{0}(\boldsymbol{x})=r^{\lambda} \cos \lambda \theta, \quad u^{2 n}(\boldsymbol{x})=(-1)^{n}\left(r^{\lambda}-r^{-\lambda}\right) \cos \lambda \theta \quad \text { for all } n \geq 1 .
$$

To obtain the inner expansion, replace $r$ by $l \rho$ in (40), to get

$$
u_{l}(l \boldsymbol{y})=\frac{l^{\lambda}}{1+l^{2 \lambda}}\left(\rho^{-\lambda}+\rho^{\lambda}\right) \cos \lambda \theta .
$$

Inserting the Taylor series as before, the expansion of $u_{l}(l \boldsymbol{y})$ is given by

$$
u_{l}(l \boldsymbol{y})=\sum_{n \in \mathbb{N}}(-1)^{n} l^{(2 n+1) \lambda}\left(\rho^{-\lambda}+\rho^{\lambda}\right) \cos \lambda \theta,
$$

which corresponds to the inner expansion where the even terms vanish and the odd terms are given by

$$
v^{2 n+1}(\boldsymbol{y})=(-1)^{n}\left(\rho^{-\lambda}+\rho^{\lambda}\right) \cos \lambda \theta \text { for all } n \geq 0 .
$$

It remains to be checked that the procedure described in the previous subsections yields the same coefficients. Since $g=0, a_{0}^{i}=0$ for all $i \geq 0$ and there is no logarithmic singularity; see Remark 4. The details for the first steps of the procedure are given below.

(S1) By (33), $a_{0}^{0}=0$ and hence $u_{S}^{0}=0$.

(S2) Hence (29) becomes: $\Delta u^{0}=0$ in $\Omega_{0}, \partial u^{0} / \partial \theta=0$ on $\theta \in\{0, \omega\}, u^{0}=\cos \lambda \theta$ on $r=1$. The unique solution in $H^{1}\left(\Omega_{0}\right)$ is $u^{0}$ given by (42).

(S3) By (37), $\mathrm{b}_{1}^{1}=1$ and $\mathrm{b}_{n}^{n}=0$ for $n \neq 1$. Hence $v_{S}^{0}=0$.

(S4) Since $v_{S}^{0}=0$ and $g=0$, (31) gives $\bar{v}^{0}=0$ and hence $v^{0}=0$.

(S5) By (36), $\mathrm{a}_{n}^{n}=0$ for $n \geq 1$.

(S6) By (25), $u_{S}^{1}=0$.

(S7) By (30), $\bar{u}^{1}=0$ and hence $u^{1}=0$.

(S8) By (37), $\mathrm{b}_{n}^{n+1}=0$ for all $n$. Hence $v_{S}^{1}=\rho^{\lambda} \cos \lambda \theta$.

(S9) Hence (32) for $i=1$ becomes: $\Delta \bar{v}^{1}=0$ in $\Omega^{\infty}, \partial \bar{v}^{1} / \partial \theta=0$ on $\theta \in\{0, \omega\}$, $\partial \bar{v}^{1} / \partial \rho=-\lambda \cos \lambda \theta$ on $\rho=1$. The unique regular solution is $\bar{v}^{1}=\rho^{\lambda} \cos \lambda \theta$ and hence $v^{1}$ is given by (45).

(S10) By (36), $\mathrm{a}_{1}^{2}=1$ and $\mathrm{a}_{n}^{n+1}=0$ for $n \neq 1$.

(S11) By (25), $u_{S}^{2}=r^{-\lambda} \cos \lambda \theta$.

(S12) Hence (30) for $i=2$ becomes: $\Delta \bar{u}^{2}=0$ in $\Omega_{0}, \partial \bar{u}^{2} / \partial \theta=0$ on $\theta \in\{0, \omega\}$, $\bar{u}^{2}=-\cos \lambda \theta$ on $r=1$. The unique solution in $H^{1}\left(\Omega_{0}\right)$ is $\bar{u}^{2}=-r^{\lambda} \cos \lambda \theta$ and hence $u^{2}$ is given by (42). 


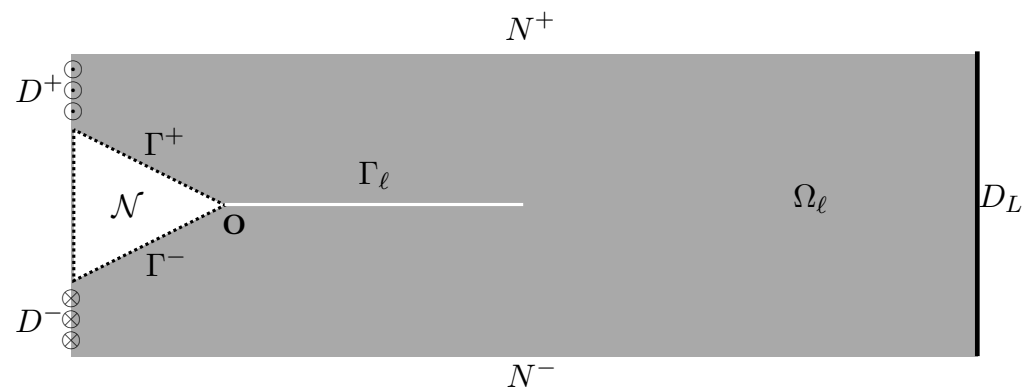

Figure 4. Definition of the cracked notch-shaped body $\Omega_{l}$ with the various parts of the boundary.

Proceeding by induction, the expected expansions are finally recovered. The end of the verification is left to the reader.

\section{Application to the case of a crack}

3.1. Setting the problem. In this section, the method is applied to a defect which is a noncohesive crack. Specifically, let $\Omega$ be the rectangle $(-H, L) \times(-H,+H)$. Let $\epsilon$ be a given parameter in $\left.(0,1), \mathcal{N}=\left\{\boldsymbol{x}=\left(x_{1}, x_{2}\right):-H<x_{1} \leq 0,\left|x_{2}\right| \leq \epsilon\left|x_{1}\right|\right)\right\}$. The notch-shaped body is $\Omega_{0}=\Omega \backslash \mathcal{N}$. Finally the cracked body $\Omega_{l}$ is obtained by removing from $\Omega_{0}$ the line segment $\Gamma_{l}=(0, l) \times\{0\}$; see Figure 4 .

The boundary $\Gamma_{D}$ where the displacement is prescribed corresponds to the sides $D^{ \pm}$and $D_{L}$, with boundary conditions

$$
u_{l}(\boldsymbol{x})=\left\{\begin{array}{cl}
+H & \text { on } D^{+}=\{-H\} \times[\epsilon H, H], \\
-H & \text { on } D^{-}=\{-H\} \times[-H,-\epsilon H], \\
0 & \text { on } D_{L}=\{L\} \times[-H, H] .
\end{array}\right.
$$

The remaining parts of the boundary (including the lips of the crack) are free; that is,

and

$$
\frac{\partial u_{l}}{\partial x_{2}}= \begin{cases}0 & \text { on } \Gamma_{l}=(0, l) \times\{0\} \\ 0 & \text { on } N^{ \pm}=(-H, L) \times\{ \pm H\}\end{cases}
$$

$$
\frac{\partial u_{l}}{\partial n}=0 \quad \text { on } \Gamma^{ \pm}=\left\{\left(x_{1}, x_{2}\right):-H<x_{1}<0, x_{2}= \pm \epsilon x_{1}\right\} .
$$

Remark 5. The amplitude of the prescribed displacement is normalized to $H$ so that $u_{l}$ has the dimension of a length. The fact that the amplitude is equal to the height $H$ has no importance in the present context of linearized elasticity. We will introduce a time-dependent amplitude of the prescribed displacement when we study the propagation of the crack. Then the prescribed displacement will take "reasonable" values, controlled by the toughness of the material. 
Remark 6. The case $\epsilon=0$ corresponds to a body with an initial crack of length $H$ and this limiting case is also considered in this paper. The case $\epsilon=1$ corresponds to a corner with an angle $\pi / 2$, the sides $D^{ \pm}$being reduced to the points $(-H, \pm H)$. This limiting case will not be considered here.

Remark 7. We only consider the case where the crack path is the line segment $(0, L) \times\{0\}$. It is a rather natural assumption by virtue of the symmetry of the geometry and the loading. An interesting extension should be to consider nonsymmetric geometry or loading and hence to take the direction of the crack as a parameter. This extension is reserved for future works.

We are in the case where $g=0$ on $\Gamma_{l}$. Therefore, by virtue of Proposition 3, all the coefficients $a_{0}^{i}$ vanish and there are no logarithmic singularities. Accordingly, the solution can be expanded as follows:

Outer expansion: $\quad u_{l}(\boldsymbol{x})=u^{0}(\boldsymbol{x})+l^{\lambda} u^{1}(\boldsymbol{x})+l^{2 \lambda} u^{2}(\boldsymbol{x})+l^{3 \lambda} u^{3}(\boldsymbol{x})+\cdots$,

Inner expansion: $\quad u_{l}(\boldsymbol{x})=v^{0}(\boldsymbol{y})+l^{\lambda} v^{1}(\boldsymbol{y})+l^{2 \lambda} v^{2}(\boldsymbol{y})+l^{3 \lambda} v^{3}(\boldsymbol{y})+\cdots$, with

$$
\lambda=\frac{\pi}{\omega} \quad \text { and } \quad \omega=2 \pi-2 \arctan (\epsilon) .
$$

By symmetry of the geometry and the loading, the real field $u_{l}$ is an odd function of $x_{2}$; i.e.,

$$
u_{l}\left(x_{1},-x_{2}\right)=-u_{l}\left(x_{1}, x_{2}\right), \quad u_{l}(r, \omega-\theta)=-u_{l}(r, \theta) .
$$

Therefore, all the fields $u^{i}, \bar{u}^{i}, v^{i}, \bar{v}^{i}$ admit the same symmetry. Therefore, by Proposition 4, all coefficients $b_{2 n}^{i+2 n}$ and $a_{2 n}^{i+2 n}$ vanish. Consequently, the odd terms of the outer expansion and the even terms of the inner expansions vanish; i.e., $u^{2 i+1}=0$ and $v^{2 i}=0$ for all $i \in \mathbb{N}$. Finally, the solution admits the following expansions:

Outer expansion: $u_{l}(\boldsymbol{x})=\sum_{i \in \mathbb{N}} l^{2 i \lambda} u^{2 i}(\boldsymbol{x})$,

Inner expansion: $\quad u_{l}(\boldsymbol{x})=\sum_{i \in \mathbb{N}} l^{(2 i+1) \lambda} v^{2 i+1}(\boldsymbol{y})$.

By symmetry, the following coefficients vanish:

$\mathrm{a}_{n}^{i}=0 \quad$ when $n$ or $i-n$ are even, $\quad \mathrm{b}_{n}^{i}=0 \quad$ when $n$ is even or $i-n$ is odd.

Examine now the singularities of $\nabla u_{l}$ (in the sense that $\nabla u_{l}$ is not bounded) according to whether or not $l=0$, and according to whether or not $\epsilon=0$. 
(1) When $\epsilon>0$ and $l=0$. Then $\nabla u_{0}$ is infinite at the tip of the notch and in its neighborhood has the form

$$
\nabla u_{0}(\boldsymbol{x})=\frac{\lambda \mathrm{b}_{1}^{1}}{r^{1-\lambda}}\left(\cos (\lambda \theta) \boldsymbol{e}_{r}-\sin (\lambda \theta) \boldsymbol{e}_{\theta}\right)+\text { regular terms }
$$

(2) When $\epsilon>0$ and $l>0$. Then $\nabla u_{l}$ is no longer infinite at the tip of the notch but becomes infinite at the tip of the crack, with the usual singularity in $1 / \sqrt{r}$; see [Bui 1978]. Specifically, $\nabla u_{l}$ has the form

$$
\nabla u_{l}(\boldsymbol{x})=\frac{K_{l}}{\mu \sqrt{2 \pi r^{\prime}}}\left(\sin \left(\frac{\theta^{\prime}}{2}\right) \boldsymbol{e}_{r}+\cos \left(\frac{\theta^{\prime}}{2}\right) \boldsymbol{e}_{\theta}\right)+\text { regular terms } .
$$

In (50), $\left(r^{\prime}, \theta^{\prime}\right)$ denotes the polar coordinate system with $\boldsymbol{x}=\left(l+r^{\prime} \cos \theta^{\prime}\right) \boldsymbol{e}_{1}+$ $r \sin \theta^{\prime} \boldsymbol{e}_{2}$ and the angular function of $\theta^{\prime}$ is normalized so that $K_{l}$ be the usual stress intensity factor. $K_{l}$ depends on $l$ and is "strongly" influenced by the presence of the notch when $l$ is small. (In fact, $K_{l}$ goes to 0 when $l$ goes to 0 as we will see below.) So, even if the stresses are only singular at the tip of the crack, there is a kind of overlapping of the previous singularity at the tip of the notch. This phenomenon renders the computations by the finite element method less accurate when $l$ is small.

(3) When $\epsilon=0$. Then the notch is already a crack and it is unnecessary to treat separately $l=0$ and $l>0$. In any case $\nabla u_{l}$ has the classical singularity in $1 \sqrt{r}$ as in (50) and there is no more overlapping of two singularities. The computations by the finite element method are accurate in the full range of values of $l$.

3.2. The issue of the computation of the energy release rate. The main goal of this section is to obtain accurate values for the elastic energy $\mathscr{P}_{l}$ stored in the cracked body and for its derivative with respect to $l$, the so-called energy release rate $\varphi_{l}$, when $l$ is small. By definition, the elastic energy is given by

$$
\mathscr{P}_{l}=\frac{1}{2} \int_{\Omega_{l}} \mu \nabla u_{l} \cdot \nabla u_{l} d x .
$$

By virtue of Clapeyron's formula, the elastic energy stored in the body when the body is at equilibrium is equal to one half the work done by the external loads over the prescribed displacement on $D^{ \pm}$. Therefore, using the symmetry of $u_{l}$, the elastic energy can also be written as an integral over $D^{+}$:

$$
\mathscr{P}_{l}=-\int_{\epsilon H}^{H} \mu H \frac{\partial u_{l}}{\partial x_{1}}\left(-H, x_{2}\right) d x_{2},
$$

which involves only the displacement field far from the tip of the notch.

By definition (see [Bourdin et al. 2008; Leblond 2003]), the energy release rate $\mathscr{G}_{l}$ is the opposite of the derivative of the elastic energy with respect to the 


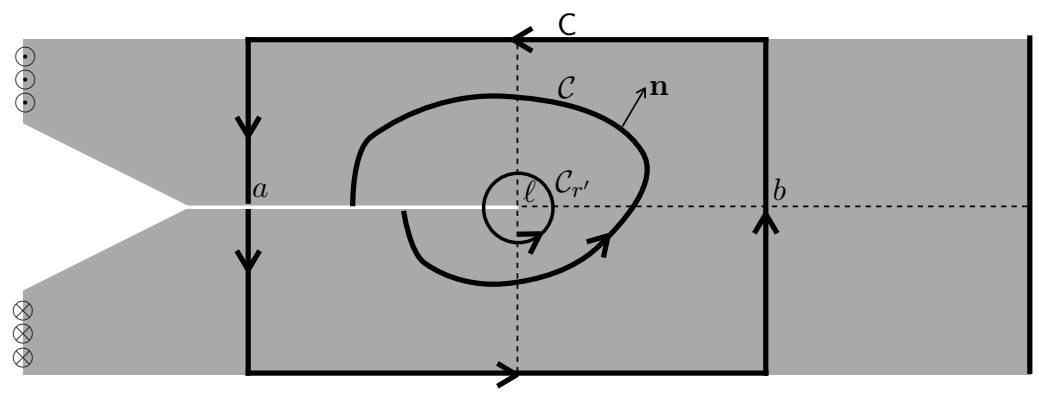

Figure 5. Examples of paths for which $\mathscr{g}_{\mathscr{C}}$ is equal to $\mathscr{G}_{l}$.

length of the crack:

$$
\varphi_{l}=-\frac{d \mathscr{P}_{l}}{d l} .
$$

Even though $\mathscr{P}_{l}$ involves the $l$-dependent displacement field $u_{l}$, its derivative does not involve the derivative $d u_{l} / d l$ but can be expressed in terms of $u_{l}$ only. This property is a consequence of the fact that $u_{l}$ satisfies the equilibrium equations. Specifically, $\mathscr{G}_{l}$ can be computed either with the help of path integrals like the $\mathscr{g}$ integral of [Rice 1968] or by using the so-called $G-\theta$ method developed in [Destuynder and Djaoua 1981]. We recall below the main ingredients of both methods when $0<l<L$. The cases $l=0$ and $l=L$ are treated separately.

In the former method, the integral $\mathscr{E}_{\mathscr{C}}$ over the path $\mathscr{C}$ is defined by

$$
\mathscr{\Phi}_{\mathscr{C}}=\int_{\mathscr{C}}\left(\frac{\mu}{2} \nabla u_{l} \cdot \nabla u_{l} n_{1}-\mu \frac{\partial u_{l}}{\partial n} \frac{\partial u_{l}}{\partial x_{1}}\right) d s,
$$

where $\boldsymbol{n}$ denotes the outer normal of the path. This integral is (theoretically) pathindependent and equal to $\mathscr{G}_{l}$ provided that the path $\mathscr{b}$ starts from the lip of the crack, circumvents the tip of the crack and finishes on the lip of the crack like in Figure 5; see [Bui 1978]. This path independence is used to obtain Irwin's formula [Irwin 1958; Leblond 2003]. Indeed, taking for path the circle $\mathscr{C}_{r^{\prime}}$ centered at the tip of the crack with radius $r^{\prime}$, using (50) and passing to the limit when $r^{\prime} \rightarrow 0$, the following link between the energy release rate and the stress intensity factor $K_{l}$ introduced in (50) is obtained:

$$
\mathscr{G}_{l}=\lim _{r^{\prime} \rightarrow 0} \mathscr{\mathscr { C }}_{\mathscr{C}_{r^{\prime}}}=\frac{K_{l}^{2}}{2 \mu} .
$$

For the computations, the particularities of the geometry and of the loading can be exploited, to choose a path made of line segments parallel to the axes like the path $\mathrm{C}$ in Figure 5:

$\mathrm{C}=\{a\} \times(-H, 0) \cup[a, b] \times\{-H\} \cup\{b\} \times(-H, H) \cup[a, b] \times\{+H\} \cup\{a\} \times(0, H)$ 
with $0<a<l<b<L$. Then $\mathscr{g}_{C}=\varphi_{l}$. Therefore, since $n_{1}=0$ and $\partial u_{l} / \partial n=0$ on the sides $x_{2}= \pm H$ and by virtue of the symmetry of $u_{l}, \mathscr{G}_{l}$ takes the form

$$
\begin{aligned}
\mathscr{G}_{l}=\mu \int_{\{b\} \times(0, H)}\left(\left(\frac{\partial u_{l}}{\partial x_{2}}\right)^{2}-\left(\frac{\partial u_{l}}{\partial x_{1}}\right)^{2}\right) d x_{2} & \\
& \quad-\mu \int_{\{a\} \times(0, H)}\left(\left(\frac{\partial u_{l}}{\partial x_{2}}\right)^{2}-\left(\frac{\partial u_{l}}{\partial x_{1}}\right)^{2}\right) d x_{2} .
\end{aligned}
$$

From a theoretical point of view, $a$ and $b$ can be chosen arbitrarily, provided that they satisfy the constraints above. Indeed, the integral over the line segment $x_{1}=a$ (respectively, $x_{1}=b$ ) does not depend on $a$ (respectively, on $b$ ) because $u_{l}$ is harmonic and satisfies homogeneous Neumann boundary conditions on $N^{ \pm}$and $\Gamma_{l}$. (This verification is left to the reader; see [Marigo 2010, Proposition 8] for a proof.) However, from a numerical point of view, this is no longer true because the computed displacement field does not satisfy exactly the equilibrium equations. Consequently, the computed values of $\varphi_{l}$ depend on the choice of $a$ and $b$. Moreover, since the integral over the line $a$ involves the gradient of the displacement, this integral can be badly approximated when $l$ is small because of the singularity.

The $G-\theta$ method is based on a change of variables which sends the $l$-dependent domain $\Omega_{l}$ onto a fixed domain. In essence, it is the basic method to prove that $l \mapsto \mathscr{P}_{l}$ is differentiable; see [Destuynder and Djaoua 1981] for the genesis of this method and [Chambolle et al. 2010] for a discussion on a generalization of the concept of energy release rate. In turn the $G-\theta$ approach gives a practical method to compute the energy release rate; see the previous two references. Specifically, for a given $l>0$, we associate to a Lipschitz continuous vector field $\boldsymbol{\theta}$ defined on $\Omega_{l}$ the volume integral

$$
\mathrm{G}_{\boldsymbol{\theta}}=\int_{\Omega_{l}}\left(\sum_{i, j=1}^{2} \mu \frac{\partial \theta_{i}}{\partial x_{j}} \frac{\partial u_{l}}{\partial x_{i}} \frac{\partial u_{l}}{\partial x_{j}}-\frac{\mu}{2} \nabla u_{l} \cdot \nabla u_{l} \operatorname{div} \boldsymbol{\theta}\right) d x
$$

It can shown that, if $\boldsymbol{\theta}$ is such that $\boldsymbol{\theta}(l, 0)=\boldsymbol{e}_{1}$ and $\boldsymbol{\theta} \cdot \boldsymbol{n}=0$ on $\partial \Omega_{l}$, then $\mathrm{G}_{\boldsymbol{\theta}}$ is independent of $\boldsymbol{\theta}$ and equal to $\mathscr{G}_{l}$. Of course, this result of independence holds only when $u_{l}$ is the true displacement field. If it is numerically approximated, then $\mathrm{G}_{\theta}$ becomes $\boldsymbol{\theta}$ dependent. In our case, owing to the simplicity of the geometry, we can use a very simple vector field $\boldsymbol{\theta}$ which renders the computations easier. Specifically, let $\boldsymbol{\theta}$ be given by

$$
\boldsymbol{\theta}(\boldsymbol{x})=\left\{\begin{array}{cl}
\boldsymbol{\Gamma} & \text { if } x_{1}<0, \\
\frac{x_{1}}{l} \boldsymbol{e}_{1} & \text { if } 0 \leq x_{1} \leq l, \\
\frac{L-x_{1}}{L-l} \boldsymbol{e}_{1} & \text { if } l \leq x_{1}<L .
\end{array}\right.
$$


It satisfies the required conditions and hence $\mathrm{G}_{\theta}=\mathscr{G}_{l}$. Accordingly, owing to the symmetry, $\mathscr{G}_{l}$ takes the form

$$
\begin{aligned}
\mathscr{\varphi}_{l}=\frac{\mu}{L-l} \int_{l}^{L} \int_{0}^{H}\left(\left(\frac{\partial u_{l}}{\partial x_{2}}\right)^{2}\right. & \left.-\left(\frac{\partial u_{l}}{\partial x_{1}}\right)^{2}\right) d x_{2} d x_{1} \\
& -\frac{\mu}{l} \int_{0}^{l} \int_{0}^{H}\left(\left(\frac{\partial u_{l}}{\partial x_{2}}\right)^{2}-\left(\frac{\partial u_{l}}{\partial x_{1}}\right)^{2}\right) d x_{2} d x_{1} .
\end{aligned}
$$

Comparing (56) with (54), (56) can be seen as an average of all the line integrals appearing in (54) when $a$ and $b$ vary, respectively, from 0 to $l$ and from $l$ to $L$. Accordingly, it can be expected that (56) gives more accurate computations than (54) when $l$ is small.

3.3. Numerical results obtained for $\mathscr{G}_{l}$ by the FEM. All the computations based on the finite element method are implemented in the industrial code COMSOL. They are performed after introducing dimensionless quantities. Specifically, in all the computations, the dimensions of the body are $H=1$ and $L=5$, the shear modulus $\mu=1$. That does not restrict the generality of the study because the scale dependencies are known in advance. Indeed, the true physical quantities are related to the normalized quantities (denoted with a tilde) by

$$
l=H \tilde{l}, \quad u_{l}=H \tilde{u}_{l}, \quad \mathscr{P}_{l}=\mu H^{2} \tilde{\mathscr{P}}_{l}, \quad \varphi_{l}=\mu H \tilde{\mathscr{G}}_{l} .
$$

For a given $\tilde{l} \in(0,5)$ and a given $\epsilon \in(0,1)$, we use the symmetry of the body and of the load to mesh only its upper half and prescribe $\tilde{u}_{l}=0$ on the segment $\tilde{l} \leq \tilde{x}_{1} \leq 5$, $\tilde{x}_{2}=0$. We use 6-node triangular elements, i.e., quadratic Lagrange interpolations. The mesh is refined near the singular corners and a typical mesh contains 25000 elements and 50000 degrees of freedom. We compute the discretized solution (still denoted) $\tilde{u}_{l}$ by solving the linear system. Then, the energy $\tilde{\mathscr{P}}_{l}$ and the energy release rate $\tilde{\mathscr{G}}_{l}$ are obtained by postprocessing. The energy is obtained by a direct integration of the elastic energy density over the body. The derivative of the energy is obtained by using formula (56), which needs to integrate the different parts of the elastic energy density over the two rectangles $(0, \tilde{l}) \times(0,1)$ and $(\tilde{l}, 5) \times(0,1)$. For a given $\epsilon$, we compute $\tilde{\mathscr{P}}_{l}$ and $\tilde{\mathscr{G}}_{l}$ for $\tilde{l}$ varying from 0.001 to 5 , first by steps of 0.001 in the interval $(0,0.05)$, then by steps of 0.002 in the interval $(0.05,0.2)$, finally by steps of 0.01 in the interval $(0.2,5)$. The computations can be considered sufficiently accurate for $\tilde{l} \geq 0.002$, even if this lower bound depends on $\epsilon$, the computations being less accurate for small (but nonzero) values of $\epsilon$. Below this value, if we try to refine the mesh near the corner of the notch, the results become mesh-sensitive, and the linear system becomes ill-conditioned. Since only the part of the graph of $\tilde{\mathscr{G}}_{l}$ close to $\tilde{l}=0$ is interesting when $\epsilon$ is small, we cannot obtain 


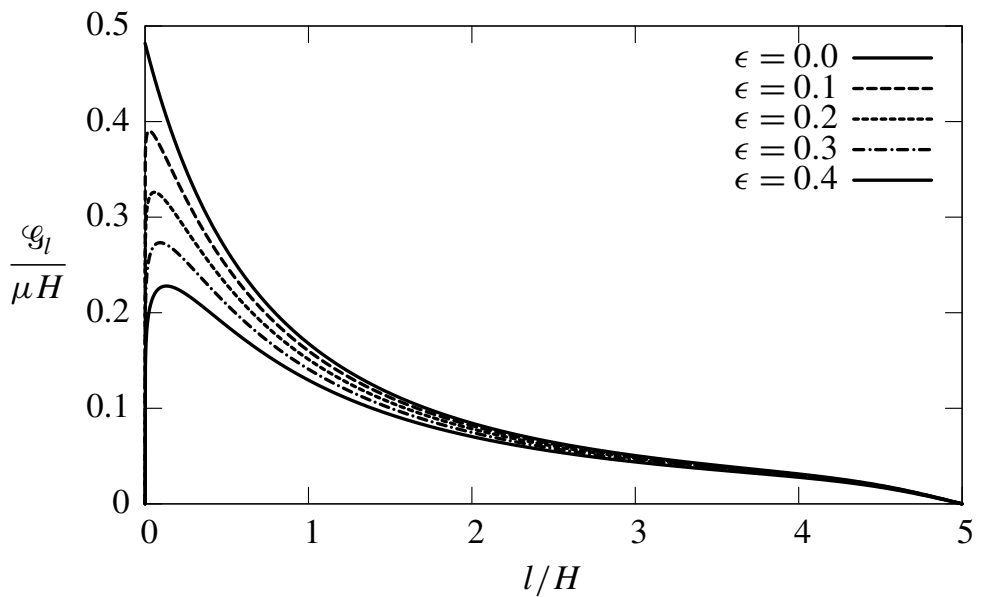

Figure 6. Computation by the Finite Element Method of the energy release rate $\varphi_{l}$ as a function of the crack length $l$ for five values of the notch angle.

accurate results when $\epsilon$ is too small. (Of course, this remark does not apply when $\epsilon=0$, because $\tilde{l}=0$ is not a "singular" case.)

The cases $\tilde{l}=0$ and $\tilde{l}=5$ with $\epsilon \neq 0$ are treated with specific meshes. We have only to compute $\tilde{u}_{0}, \tilde{\mathscr{P}}_{0}, \tilde{u}_{L}$ and $\tilde{\mathscr{P}}_{L}$, since $\tilde{\varphi}_{0}=\tilde{\mathscr{G}}_{L}=0$.

The case $\epsilon=0$ is treated separately by adapting the previous methods. In particular, to calculate $\tilde{\mathscr{G}}_{l}$, the second integral in (56) is replaced by an integral over the rectangle $(-1,0) \times(0,1)$, and this integral is divided by $1+\tilde{l}$ instead of $\tilde{l}$. Moreover, the mesh is refined only near the tip of the crack; $\tilde{l}=0$ is no longer a particular case and the computations of $\tilde{\varphi}_{l}$ are accurate in the full range of $\tilde{l}$.

Let us highlight the main features of the numerical results plotted in Figure 6. These properties will be the basic assumptions from which we study the crack propagation at the end of the present section.

(P1) For $\epsilon=0, \mathscr{G}_{l} / \mu H$ is monotonically decreasing from 0.4820 to 0 when $l / H$ grows from 0 to 5 .

(P2) For $\epsilon>0, \mathscr{G}_{l} / \mu H$ starts from 0 at $l / H=0$, then is rapidly increasing. This growth is of such magnitude (for instance, $\mathscr{G}_{l} / \mu H=0.1443$ when $l / H=$ 0.002 for $\epsilon=0.4$ ) that it cannot be correctly captured by the FEM.

(P3) Still for $\epsilon>0, \mathscr{G}_{l}$ is monotonically increasing as long as $l \leq l_{\mathrm{m}}$. At $l=l_{\mathrm{m}}, \mathscr{G}$ takes its maximal value $G_{m}$. Those values which depend on $\epsilon$ are given in the table below. It turns out that $l_{\mathrm{m}} / H$ is rather small. 


\begin{tabular}{|c|ccccc|}
\hline$\epsilon$ & 0 & 0.1 & \multicolumn{1}{c}{0.2} & \multicolumn{1}{c}{0.3} & 0.4 \\
\hline$l_{\mathrm{m}} / H$ & 0 & 0.024 & 0.058 & 0.092 & 0.130 \\
$\mathrm{G}_{\mathrm{m}} / \mu H$ & 0.4820 & 0.3900 & 0.3260 & 0.2733 & 0.2279 \\
\hline
\end{tabular}

(P4) For $\epsilon>0$ again, $\mathscr{G}_{l}$ is monotonically decreasing from $\mathrm{G}_{\mathrm{m}}$ to 0 when $l$ grows from $l_{\mathrm{m}}$ to $5 H$.

3.4. Evaluation of the energy release rate by the MAM. By virtue of (52), $\mathscr{P}_{l}$ can be expanded by using the outer expansion of $u_{l}$. Using (47) leads to

$$
\mathscr{P}_{l}=\sum_{i \in \mathbb{N}} P_{2 i}\left(\frac{l}{H}\right)^{2 i \lambda} \mu H^{2},
$$

where the coefficients $P_{2 i}$ of the expansions are dimensionless. The expansion of the energy release rate can be immediately deduced from that of the energy:

$$
\mathscr{G}_{l}=-\sum_{i \in \mathbb{N}^{*}} 2 i \lambda P_{2 i}\left(\frac{l}{H}\right)^{2 i \lambda-1} \mu H,
$$

and it is not necessary to use the path integrals $\mathscr{F}_{\mathscr{C}}$ or the $G-\theta$ method. Let us remark that

$$
\mathscr{G}_{0}=\left\{\begin{array}{cc}
0 & \text { if } \epsilon \neq 0, \\
-P_{2} \mu H=K_{0}^{2} / 2>0 & \text { if } \epsilon=0,
\end{array}\right.
$$

because $\lambda>1 / 2$ in the former case while $\lambda=1 / 2$ in the latter.

To obtain the $i$-th term of the expansion of $\mathscr{P}_{l}$ and $\mathscr{G}_{l}$, both the singular part $u_{S}^{i}$ and the regular part $\bar{u}^{i}$ of $u^{i}$ must be recovered. The singular part involves the coefficients $\mathrm{a}_{n}^{i}$ for $1 \leq n \leq i$ which are obtained as the regular parts of the $v^{j}$ for $j \leq i$; see Section 2.2.6. Therefore, the inner problems must be solved to determine the coefficients $\mathrm{b}_{n}^{i}$ for $0 \leq n \leq i$. In practice, these coefficients are obtained by using Proposition 4 after the inner and the outer problems have been solved with a finite element method. The advantage is that those problems do not contain a small defect and the accuracy is guaranteed. The drawback is that more and more problems have to be solved, in order to obtain accurate values of $\varphi_{l}$ when $l / H$ is not small.

In Tables 3 and 4 are given the computed values of the first coefficients of the inner and outer expansions (still with $H=1, L=5, \mu=1$ ). These tables contain all the terms which are necessary to compute the expansions of the energy up to the sixth order, i.e., $P_{2 i}$ for $i \in\{0,1,2,3\}$. (Note that $P_{0}$ does not appear in the expansion of $\varphi_{l}$.) The graphs of $l \mapsto \mathscr{G}_{l}$ obtained from these expansions are plotted in Figure 7 in the cases $\epsilon=0.2$ and $\epsilon=0.4$. They are compared with the values obtained directly by the finite element code COMSOL. From these comparisons, the following conclusions can be drawn: 


\begin{tabular}{cccccccccc}
$\epsilon$ & $\mathrm{a}_{1}^{2}$ & $P_{2}$ & $\mathrm{a}_{1}^{4}$ & $\mathrm{a}_{3}^{4}$ & $P_{4}$ & $\mathrm{a}_{1}^{6}$ & $\mathrm{a}_{3}^{6}$ & $\mathrm{a}_{5}^{6}$ & $P_{6}$ \\
\hline 0 & -0.3930 & -0.4820 & 0.1888 & 0.0987 & 0.3282 & -0.1365 & -0.0537 & -0.0494 & -0.2013 \\
0.1 & -0.3756 & -0.4413 & 0.1766 & 0.0943 & 0.3001 & -0.1279 & -0.0507 & -0.0472 & -0.1931 \\
0.2 & -0.3559 & -0.3957 & 0.1619 & 0.0893 & 0.2673 & -0.1165 & -0.0470 & -0.0446 & -0.1787 \\
0.3 & -0.3342 & -0.3486 & 0.1453 & 0.0838 & 0.2320 & -0.1029 & -0.0427 & -0.0418 & -0.1603 \\
0.4 & -0.3106 & -0.3005 & 0.1273 & 0.0778 & 0.1952 & -0.0880 & -0.0380 & -0.0389 & -0.1385 \\
\hline
\end{tabular}

Table 3. The computed values of the (nonzero) coefficients $a_{n}^{i}$ for $1 \leq n \leq i \leq 6$ and of the leading terms $P_{2}, P_{4}$ and $P_{6}$ of the expansion of the potential energy for several values of the angle of the notch.

\begin{tabular}{ccccccc}
$\epsilon$ & $\mathrm{b}_{1}^{1}$ & $\mathrm{~b}_{1}^{3}$ & $\mathrm{~b}_{3}^{3}$ & $\mathrm{~b}_{1}^{5}$ & $\mathrm{~b}_{3}^{5}$ & $\mathrm{~b}_{5}^{5}$ \\
\hline 0 & -0.7834 & 0.2384 & -0.2059 & -0.1943 & 0.1058 & -0.0172 \\
0.1 & -0.7482 & 0.2091 & -0.2085 & -0.1730 & 0.0992 & -0.0283 \\
0.2 & -0.7089 & 0.1777 & -0.2081 & -0.1489 & 0.0905 & -0.0379 \\
0.3 & -0.6657 & 0.1451 & -0.2045 & -0.1232 & 0.0800 & -0.0454 \\
0.4 & -0.6187 & 0.1125 & -0.1977 & -0.0974 & 0.0683 & -0.0508 \\
\hline
\end{tabular}

Table 4. The computed values of the (nonzero) coefficients $\mathrm{b}_{n}^{i}$ for $1 \leq n \leq i \leq 5$ for several values of the angle of the notch.

(C1) For very small values of $l$, the first nontrivial term (corresponding to $i=1$ in (59)) of the matched asymptotic expansion (denoted by MAM 2 in Figure 7) is sufficient to well approximate $\varphi_{l}$ while the FEM is unable to deliver accurate values.

(C2) For values of $l$ of the order of $l_{\mathrm{m}}$, at least the first two nontrivial terms (corresponding to $i=1$ and 2 in (59)) of the MAE (denoted by MAM 4 in Figure 7) are necessary to capture the change of monotonicity of $\mathscr{G}_{l}$. Indeed, the first term, being monotonically increasing, is unable, alone, to capture that change of behavior.

(C3) Still for values of $l$ of the order of $l_{\mathrm{m}}$, the first two terms are really sufficient to well approximate $\varphi_{l}$ provided that $l_{\mathrm{m}} / H$ is sufficiently small. Specifically, the first two terms are sufficient as long as $l / H<0.2$.

(C4) Accordingly, the approximation of $\varphi_{l}$ by the first two nontrivial terms of the MAE can be used, in the range $\left[0,2 l_{\mathrm{m}}\right]$ of $l$ when $\epsilon \in(0,0.4)$.

(C5) As $l / H$ grows beyond 0.2, more and more terms of the MAE must be added, in order to get a good approximation of $\mathscr{G}_{l}$. Consequently, in the range of 
"large" values of $l / H$, the direct FEM is more accurate and hence is better to use.

\section{Application to the determination of the nucleation of the crack}

The theoretical and numerical results obtained in the previous sections are used here to study the delicate issue of the nucleation of a crack in a sound body or the most classical question of the onset of a preexisting crack. Specifically, we consider the notched body $\Omega_{0}$ which either contains a preexisting crack $l_{0}>0$ or is sound; i.e., $l_{0}=0$. We have also to distinguish different cases according to whether $\epsilon=0$ or $\epsilon>0$. The nucleation or the onset of cracking is governed by either the so-called G-law or the so-called FM-law and one goal of this section is to compare those laws. The interested reader can also refer to [Bourdin et al. 2008; Francfort and Marigo 1998; Negri 2010; Negri and Ortner 2008; Marigo 2010] where other comparisons between the G-law and the FM-law are proposed.

The notched body is submitted to a time-dependent loading process which consists of a monotonically increasing amplitude of the displacement prescribed on the sides $D^{ \pm}$. Specifically, consider the new boundary conditions

$$
u= \pm t H \quad \text { on } D^{ \pm}, \quad t \geq 0 .
$$

The others remain unchanged. (Note that the "time" parameter $t$ is dimensionless.) The evolution problem consists of finding the time evolution of the length of the crack, i.e., $t \mapsto l(t)$ for $t \geq 0$, under the initial condition $l(0)=l_{0} \in[0, L)$. For that, we first remark that, for a given time $t \geq 0$ and a given crack length $l \in[0, L]$, the displacement field which equilibrates the body is

$$
u(t, l)=t u_{l},
$$

where $u_{l}$ is the displacement field introduced in Section 3.1. Accordingly, the potential energy and the energy release rate at time $t$ with a crack length $l$ can be expressed as

$$
\mathscr{P}(t, l)=t^{2} \mathscr{P}_{l}, \quad \mathscr{G}(t, l)=t^{2} \mathscr{G}_{l}
$$

where $\mathscr{P}_{l}$ and $\varphi_{l}$ are given by (51) and (53).

The two evolution laws are based on Griffith's crucial assumption [1921] concerning the surface energy associated with a crack. Specifically, assume that there exists a material constant $G_{c}>0$ such that the surface energy of the body with a crack of length $l$ is

$$
\mathscr{S}(l)=\mathrm{G}_{\mathrm{c}} l .
$$



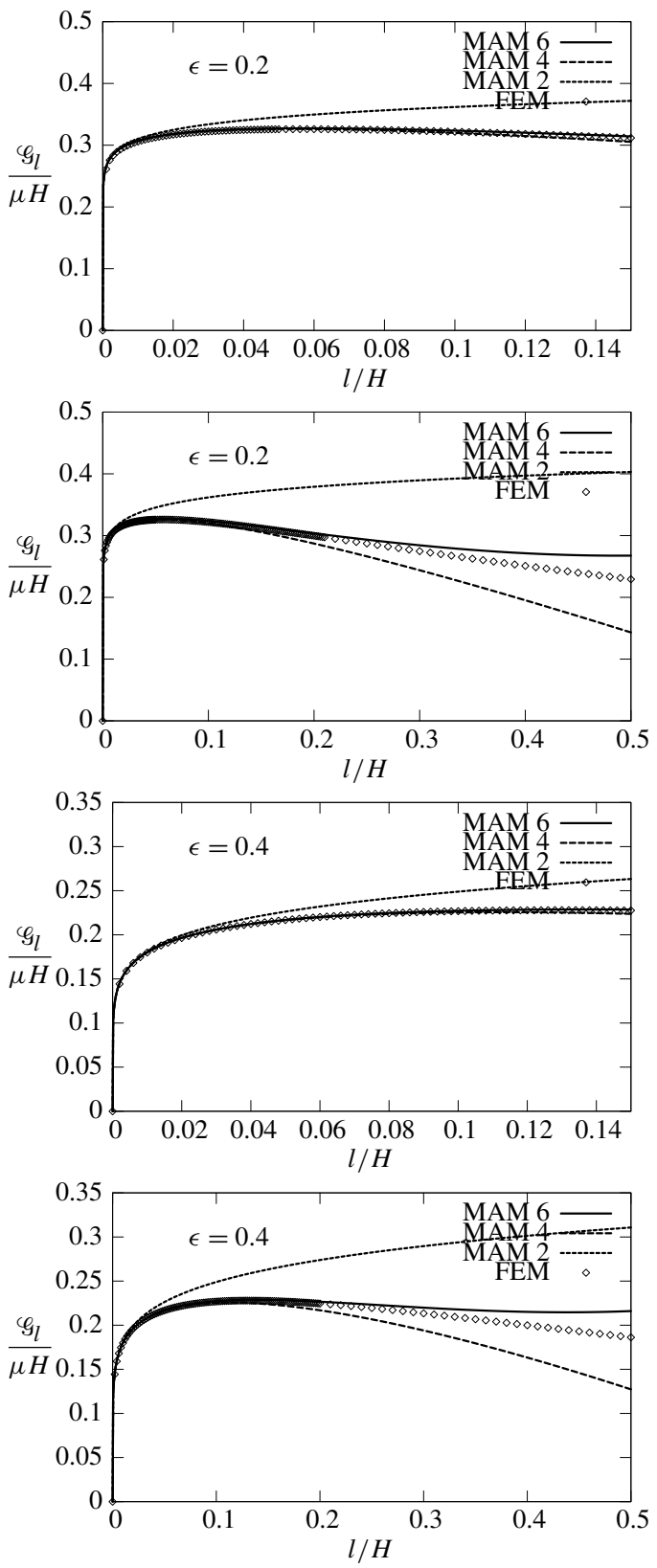

Figure 7. Comparison of the graphs of $\varphi_{l}$ obtained by the MAM and by COMSOL for $\epsilon=0.2$ (top two plots) and $\epsilon=0.4$ (bottom two). The curve labeled FEM indicates points obtained by COMSOL, while the curves labeled MAM $2 i, i \in\{1,2,3\}$, indicate that the first $i$ nontrivial terms in the expansion of $\mathscr{G}_{l}$ were considered. 
Accordingly, the total energy of the body at equilibrium at time $t$ with a crack of length $l$ becomes

$$
\mathscr{E}(t, l):=\mathscr{P}(t, l)+\mathscr{S}(l)=t^{2} \mathscr{P}_{l}+\mathrm{G}_{c} l .
$$

Throughout this section we assume that $l \mapsto \mathscr{P}_{l}$ is continuously differentiable and monotonically decreasing. Moreover, some monotonic properties of $l \mapsto \varphi_{l}$ will be added when necessary according to the analysis made in the previous sections.

4.1. The two evolution laws. Let us briefly introduce the two evolution laws; the reader interested in the details should refer to [Marigo 2010]. The first one, called the G-law, is the usual Griffith law based on the critical potential energy release rate criterion; see [Bui 1978; Leblond 2003; Nguyen 2000]. In essence, this law only investigates smooth (i.e., at least continuous) evolutions of the crack length with the loading. It consists of the three following items:

Definition $2\left(G\right.$-law). Let $l_{0} \in[0, L]$. A continuous function $t \mapsto l(t)$ is said to satisfy (or to be a solution of) the $G$-law in the interval $\left[t_{0}, t_{1}\right]$ with the initial condition $l\left(t_{0}\right)=l_{0}$, if the three following properties hold:

(1) Irreversibility: $t \mapsto l(t)$ is not decreasing;

(2) Energy release rate criterion: $\mathscr{G}(t, l(t)) \leq \mathrm{G}_{\mathrm{c}}$ for all $t \in\left[t_{0}, t_{1}\right]$;

(3) Energy balance: $l(t)$ is increasing only if $\mathscr{G}(t, l(t))=\mathrm{G}_{\mathrm{c}}$; i.e., if $\mathscr{G}(t, l(t))<$ $\mathrm{G}_{\mathrm{c}}$ at some $t$, then $l\left(t^{\prime}\right)=l(t)$ for every $t^{\prime}$ in a certain neighborhood $[t, t+h)$ of $t$.

The third item implies that the release of potential energy is equal to the created surface energy when the crack propagates, which justifies its name "energy balance". Consequently, if $t \mapsto l(t)$ is absolutely continuous, then the third item is equivalent to

$$
\frac{\partial \mathscr{E}}{\partial l}(t, l(t)) \dot{l}(t)=0
$$

for almost all $t$, and the following equality holds for almost all $t$ :

$$
\frac{d}{d t} \mathscr{E}(t, l(t))=\frac{\partial \mathscr{E}}{\partial t}(t, l(t)) .
$$

A major drawback of the G-law is the inability to take into account discontinuous crack evolutions, which renders it useless in many situations as we will see in the next subsection. It must be replaced by another law which admits discontinuous solutions. Another motivation of changing the G-law is to reinforce the second item by introducing a full stability criterion; see [Francfort and Marigo 1998; Nguyen 2000; Bourdin et al. 2008]. Specifically, let us consider the local stability condition

$$
\forall t \geq 0, \exists h(t)>0: \quad \mathscr{E}(t, l(t)) \leq \mathscr{E}(t, l) \quad \forall l \in[l(t), l(t)+h(t)],
$$


which requires that the total energy at $t$ is a "unilateral" local minimum. (The qualifier unilateral is added because the irreversibility condition leads to comparing the energy at $t$ with only that corresponding to greater crack length; see [Bourdin et al. 2008].) Taking $l=l(t)+h$ with $h>0$ in (67), dividing by $h$ and passing to the limit when $h \rightarrow 0$, we recover the critical energy release rate criterion. Thus, the second item can be seen as a first-order stability condition, weaker than (67). A stronger requirement is obtained by replacing local minimality by global minimality. It was the condition introduced in [Francfort and Marigo 1998] in the spirit of the original Griffith idea [1921], and we will adopt it here.

Definition 3 (FM-law). A function $t \mapsto l(t)$ (defined for $t \geq 0$ and with values in $[0, L]$ ) is said to satisfy (or to be a solution of) the FM-law if the three following properties hold:

(1) Irreversibility: $t \mapsto l(t)$ is not decreasing;

(2) Global stability: $\mathscr{E}(t, l(t)) \leq \mathscr{E}(t, l)$ for all $t \geq 0$ and all $l \in[l(t), L]$;

(3) Energy balance: $\mathscr{E}(t, l(t))=\mathscr{E}\left(0, l_{0}\right)+\int_{0}^{t} \partial \mathscr{E} / \partial t^{\prime}\left(t^{\prime}, l\left(t^{\prime}\right)\right) d t^{\prime}$ for all $t \geq 0$.

Let us note that the irreversibility condition is unchanged, while the energy balance condition is now written as the integrated form of (66), which does not require that $t \mapsto l(t)$ be continuous. Note also that the energy balance implies $l(0)=l_{0}$ because $0=\mathscr{E}(0, l(0))-\mathscr{E}\left(0, l_{0}\right)=\mathrm{G}_{\mathrm{c}}\left(l(0)-l_{0}\right)$, and that the second item is automatically satisfied at $t=0$ because $\mathscr{E}(0, l)=\mathrm{G}_{c} l$.

4.2. The main properties of the G-law and the FM-law. We recall or establish in this subsection some results for the two evolution laws under the assumptions of monotonicity of $l \mapsto \varphi_{l}$ resulting from the numerical computations; see (P1)(P4) in Section 3.3. Some of those results have a general character and have been previously established in [Bourdin et al. 2008; Francfort and Marigo 1998; Marigo 2010], while the other ones are specific to the present problem. In the case of properties which have already been obtained, we simply recall them without proofs.

Let us first consider the case when the notch is in fact a crack. Then, the two laws are equivalent by virtue of:

Proposition 5. In the case $\epsilon=0$, since $l \mapsto \mathscr{G}_{l}$ is decreasing from $\mathscr{G}_{0}>0$ to 0 when $l$ goes from 0 to $L$ (see property $(\mathbf{P 1})$ ), the G-law and the FM-law admit the same unique solution. Specifically, the preexisting crack begins to propagate at time $\mathrm{t}_{\mathrm{i}}$ such that $\mathrm{t}_{\mathrm{i}}{ }^{2} \varphi_{l_{0}}=\mathrm{G}_{\mathrm{c}}$. Then the crack propagates continuously and $l(t)$ is such that $t^{2} \varphi_{l(t)}=G_{c}$. Since $\varphi_{L}=0$, the crack will not reach the end $L$ in a finite time.

Proof. See [Marigo 2010, Proposition 18].

In the case of a genuine notch, as far as the nucleation and the propagation of a crack with the G-law are concerned, we have: 
Proposition 6. In the case $\epsilon>0$, according to $l_{0}=0$ or $l_{0} \in\left(0, l_{\mathrm{m}}\right)$ or $l_{0} \in\left[l_{\mathrm{m}}, L\right)$, the crack evolution predicted by the G-law is as follows:

(1) If $l_{0}=0$, since $\mathscr{G}_{0}=0$, the unique solution to the G-law is $l(t)=0$ for all $t$; i.e., there is no crack nucleation.

(2) If $l_{0} \in\left(0, l_{\mathrm{m}}\right)$, then the preexisting crack begins to propagate at time $\mathrm{t}_{\mathrm{i}}$ such that $\mathrm{t}_{\mathrm{i}}^{2} \varphi_{l_{0}}=\mathrm{G}_{\mathrm{c}}$. But at $\mathrm{t}_{\mathrm{i}}$ the propagation is necessarily discontinuous and hence there is no continuous solution to the G-law for $t \geq \mathrm{t}_{\mathrm{i}}$.

(3) If $l_{0} \in\left[l_{\mathrm{m}}, L\right)$, since $l \mapsto \mathscr{G}_{l}$ is monotonically decreasing in the interval $\left(l_{\mathrm{m}}, L\right)$, the situation is the same as in Proposition 5. There exists a unique solution for the G-law: the crack begins to propagate at $\mathrm{t}_{\mathrm{i}}$ (still given by $\mathrm{t}_{\mathrm{i}}^{2} \varphi_{l_{0}}=\mathrm{G}_{\mathrm{c}}$ ) and then propagates continuously until L, which is reached asymptotically.

Proof. Let us give the sketch of the proof for the first two items.

(1) Since $l_{0}=0$ and $\mathscr{G}_{0}=0$, then for all $t \geq 0$ one gets $0=\mathscr{G}(t, 0)<\mathrm{G}_{\mathrm{c}}$ and hence $l(t)=0$ is a solution. The uniqueness follows from the initial condition and the energy balance.

(2) Since $0<l_{0}<l_{\mathrm{m}}$, then $\mathscr{G}_{l_{0}}>0$ and hence $t^{2} \mathscr{G}_{l_{0}}=\mathscr{G}\left(t, l_{0}\right) \leq \mathrm{G}_{\mathrm{c}}$ if and only if $t \in\left[0, \mathrm{t}_{\mathrm{i}}\right]$. Since the inequality is strict when $t \in\left[0, \mathrm{t}_{\mathrm{i}}\right)$, then $l(t)=0$ is the unique solution in this interval because of the initial condition and the energy balance. By continuity, it is also the unique solution in the closed interval $\left[0, \mathrm{t}_{\mathrm{i}}\right]$. On the other hand, since $\mathscr{G}\left(t, l_{0}\right)>\mathrm{G}_{\mathrm{c}}$ when $t>\mathrm{t}_{\mathrm{i}}$, the crack must begin to propagate at $t_{i}$.

Let us show that no (continuous) evolution can satisfy the G-law for $t>\mathrm{t}_{\mathrm{i}}$. Indeed, by construction $\mathscr{G}\left(\mathrm{t}_{\mathrm{i}}, l\left(\mathrm{t}_{\mathrm{i}}\right)\right)=\mathrm{t}_{\mathrm{i}}^{2} \mathscr{G}_{l_{0}}=\mathrm{G}_{\mathrm{c}}$. But since $l(t) \geq l_{\mathrm{i}}$ for $t>\mathrm{t}_{\mathrm{i}}$ and since $l \mapsto \mathscr{G}_{l}$ is monotonically increasing in the neighborhood of $l_{0}<l_{\mathrm{m}}$, we have for $t \in\left(\mathrm{t}_{\mathrm{i}}, \mathrm{t}_{\mathrm{i}}+h\right)$ and a sufficiently small $h>0$ :

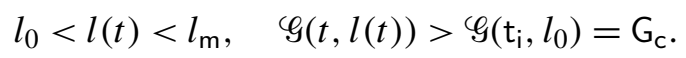

Therefore the energy release rate criterion cannot be satisfied by a continuous evolution in a neighborhood of $t_{i}$. The unique possibility is that the length of the crack jumps from $l_{0}$ to some $l_{\mathrm{i}}>l_{\mathrm{m}}$ at time $\mathrm{t}_{\mathrm{i}}$. But that requires reformulating the G-law.

The proof of the third item is the same as in the previous proposition and hence we refer to [Marigo 2010, Proposition 18].

Remark 8. This property of no nucleation of a crack at a notch or of brutal propagation of a short crack is due to the fact that a notch with Neumann boundary conditions induces a weak singularity only; i.e., $\lambda>1 / 2$. If one changes the boundary conditions by imposing the displacement on one edge of the notch and the stress on the other edge, then the singularity becomes strong for $\omega$ large enough 
and in such a case all the properties of nucleation are changed; see [Francfort and Marigo 1998, Proposition 4.19].

Consider now the FM-law. It is proved in [Marigo 2010, Proposition 3] that, in the case of a monotonically increasing loading, the FM-law is equivalent to a minimization problem of the total energy at each time, as precisely stated in the following lemma:

Lemma 7. Let $l_{0} \in[0, L)$ be the initial length of the crack. A function $t \mapsto l(t)$ satisfies the FM-law if and only if, at each $t, l(t)$ is a minimizer of $l \mapsto \mathscr{E}(t, l)$ over $\left[l_{0}, L\right]$. Therefore, the FM-law admits at least one solution and each solution grows from $l_{0}$ to $L$.

This property holds true for any $\epsilon \geq 0$. In the case $\epsilon>0$ we can deduce precise results:

Proposition 8. In the case $\epsilon>0$, according to $l_{0} \in\left[0, l_{\mathrm{m}}\right)$ or $l_{0} \in\left[l_{\mathrm{m}}, L\right)$, the crack evolution predicted by the FM-law is as follows:

(1) If $l_{0} \in\left[0, l_{\mathrm{m}}\right.$ ), then the nucleation (if $l_{0}=0$ ) or the propagation of the preexisting crack (if $\left.l_{0} \neq 0\right)$ starts at time $\mathrm{t}_{\mathrm{i}}>0$ and at this time the crack length jumps instantaneously from $l_{0}$ to $l_{\mathrm{i}}$. The length $l_{\mathrm{i}}$ is the unique length in $\left(l_{\mathrm{m}}, L\right)$ such that

$\int_{l_{0}}^{l_{\mathrm{i}}} \mathscr{G}_{l} d l=\left(l_{\mathrm{i}}-l_{0}\right) \mathscr{G}_{l_{\mathrm{i}}}, \quad$ or, equivalently, $\quad \mathscr{P}_{l_{0}}-\mathscr{P}_{l_{\mathrm{i}}}=\left(l_{\mathrm{i}}-l_{0}\right) \mathscr{G}_{l_{\mathrm{i}}}$,

while the time $\mathrm{t}_{\mathrm{i}}$ is given by

$$
\mathrm{t}_{\mathrm{i}}^{2} \varphi_{l_{\mathrm{i}}}=\mathrm{G}_{\mathrm{c}} .
$$

After this jump, the crack propagates continuously from $l_{\mathrm{i}}$ to $L$, the evolution satisfying then the G-law; i.e.,

$$
t^{2} \varphi_{l(t)}=\mathrm{G}_{\mathrm{c}} \text { for all } t>\mathrm{t}_{\mathrm{i}} .
$$

(2) If $l_{0} \in\left[l_{\mathrm{m}}, L\right)$, since $l \mapsto \mathscr{G}_{l}$ is monotonically decreasing in the interval $\left(l_{\mathrm{m}}, L\right)$, the situation is the same as in Proposition 5. There exists a unique solution for the FM-law which is the same as for the G-law: the crack begins to propagate at $\mathrm{t}_{\mathrm{i}}$ such that $\mathrm{t}_{\mathrm{i}}^{2} \varphi_{l_{0}}=\mathrm{G}_{\mathrm{c}}$ and then propagates continuously until L, which is reached asymptotically.

Remark 9. Before the proof of this proposition, let us comment and interpret (68) giving the jump of the crack at $t_{i}$. 


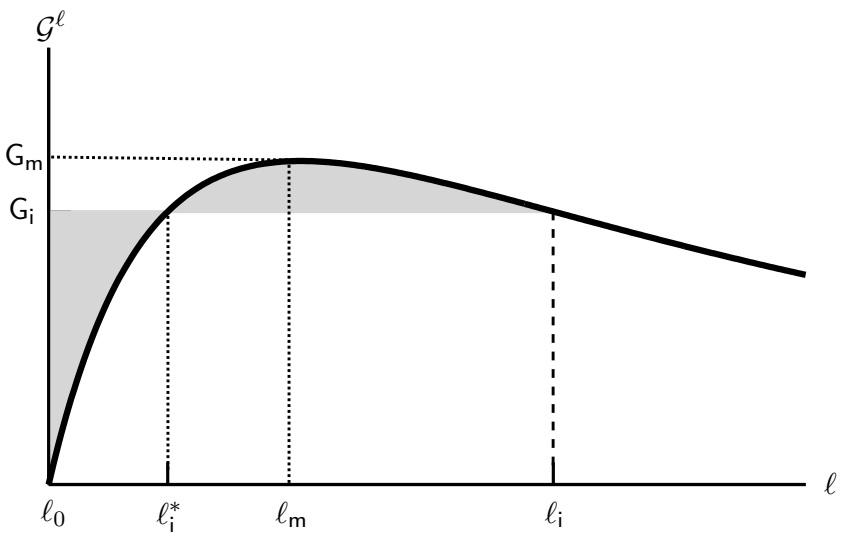

Figure 8. Graphical interpretation of the criterion of crack nucleation given by the FM-law and which obeys the Maxwell rule of equal areas.

- Let us first prove that $l_{\mathrm{i}}$ is well defined by (68). Let $l \mapsto g(l)$ be the function defined for $l \in\left(l_{\mathrm{m}}, L\right)$ by

$$
g(l)=\int_{l_{0}}^{l} \varphi_{l} d l-\left(l-l_{0}\right) \mathscr{G}_{l} .
$$

Its derivative is given by $g^{\prime}(l)=-\left(l-l_{0}\right) \mathscr{G}_{l}^{\prime}$ and hence is positive because $\mathscr{G}_{l}$ is decreasing in $\left(l_{\mathrm{m}}, L\right)$. Since $\varphi_{l}<\mathrm{G}_{\mathrm{m}}:=\mathscr{G}_{l_{\mathrm{m}}}, g\left(l_{\mathrm{m}}\right)<0$, whereas $g(L)>0$ because $\varphi_{L}=0$. Therefore, there exists a unique $l \in\left(l_{\mathrm{m}}, L\right)$ such that $g(l)=0$, what is precisely the definition of $l_{\mathrm{i}}$.

- Equation (68) giving $l_{\mathrm{i}}$ has a graphical interpretation. Indeed, the integral over $\left(l_{0}, l_{\mathrm{i}}\right)$ represents the area under the graph of $l \mapsto \mathscr{G}_{l}$ between the lengths $l_{0}$ and $l_{\mathrm{i}}$. On the other hand the product $\left(l_{\mathrm{i}}-l_{0}\right) \mathscr{G}_{l_{\mathrm{i}}}$ represents the area of the rectangle whose height is $G_{i}:=\mathscr{G}_{l_{\mathrm{i}}}$. Therefore, since these two areas are equal, the two gray areas of Figure 8 are also equal. This rule of equality of the areas determines $l_{\mathrm{i}}$ and, by essence, the line $G=\mathrm{G}_{\mathrm{i}}$ is the classical Maxwell line which appears in any problem of minimization of a nonconvex function.

- Note that $l_{\mathrm{i}}$ is independent of the toughness $\mathrm{G}_{\mathrm{c}}$ and of the shear modulus $\mu$ of the material. It is a characteristic of the structure and merely depends on the geometry and the type of loading. Here, it depends on $\epsilon, H$ and $L$. For a given $\epsilon$ and a given ratio $L / H, l_{\mathrm{i}}$ is proportional to $H, l_{\mathrm{i}}=\tilde{l}_{\mathrm{i}} H$. This property is a consequence of the Griffith assumption on the surface energy.

- The critical loading amplitude $\mathrm{t}_{\mathrm{i}}$ depends on the toughness and on the size of the body. Since $\mathscr{G}_{l_{\mathrm{i}}}=\tilde{\varphi}_{l_{\mathrm{i}}} \mu H, \mathrm{t}_{\mathrm{i}}$ varies like $1 / \sqrt{H}$. This size effect is also a consequence of the Griffith assumption on the surface energy. 
- By virtue of (68) and (69), the energy balance holds at time $t_{\mathrm{i}}$ even if the crack jumps at this time; i.e., the total energy of the body just before the jump is equal to the total energy just after. Indeed, those energies are respectively given by

$$
\mathscr{E}\left(\mathrm{t}_{\mathrm{i}}-, l_{0}\right)=\mathrm{t}_{\mathrm{i}}^{2} \mathscr{P}_{l_{0}}+\mathrm{G}_{\mathrm{c}} l_{0}, \quad \mathscr{E}\left(\mathrm{t}_{\mathrm{i}}+, l_{\mathrm{i}}\right)=\mathrm{t}_{\mathrm{i}}^{2} \mathscr{P}_{l_{\mathrm{i}}}+\mathrm{G}_{\mathrm{c}} l_{\mathrm{i}} .
$$

Using (68), (69) and the equality $\mathscr{P}_{l_{0}}-\mathscr{P}_{l_{\mathrm{i}}}=\int_{l_{0}}^{l_{\mathrm{i}}} \varphi_{l} d l$, then $\mathscr{E}\left(\mathrm{t}_{\mathrm{i}}-, l_{0}\right)=$ $\mathscr{E}\left(\mathrm{t}_{\mathrm{i}}+, l_{\mathrm{i}}\right)$.

Proof of Proposition 8. We just prove the first part of the proposition and the reader should refer to [Marigo 2010, Proposition 18] for the proof of the second part. Let $l_{0} \in\left[0, l_{\mathrm{m}}\right)$. By virtue of Lemma $7, l(t)$ is a minimizer of $l \mapsto \mathscr{E}(t, l)$ over $\left[l_{0}, L\right]$. (The minimum exists because the energy is continuous and the interval is compact.) Let $l_{\mathrm{i}}, \mathrm{t}_{\mathrm{i}}$ be given by (68)-(69), let $\mathrm{G}_{\mathrm{i}}=\mathscr{G}_{l_{\mathrm{i}}}$ and let $l_{\mathrm{i}}^{*}$ be the other length such that $\varphi_{l_{i}^{*}}=\mathrm{G}_{\mathrm{i}}$; see Figure 8. Let us first remark that the function $l \mapsto \bar{g}(l)$ defined on $\left[l_{0}, L\right]$ by

$$
\bar{g}(l):=\mathrm{G}_{\mathrm{i}}\left(l-l_{0}\right)-\left(\mathscr{P}_{l_{0}}-\mathscr{P}_{l}\right)
$$

is nonnegative and vanishes only at $l_{0}$ and $l_{\mathrm{i}}$. Indeed, its derivative is $\bar{g}^{\prime}(l)=\mathrm{G}_{\mathrm{i}}-\varphi_{l}$. Hence, $\bar{g}$ is first increasing from 0 when $l$ grows from $l_{0}$ to $l_{\mathrm{i}}^{*}$, then decreasing to 0 when $l$ grows from $l_{\mathrm{i}}^{*}$ to $l_{\mathrm{i}}$, and finally increasing again from 0 when $l$ grows from $l_{\mathrm{i}}$ to $L$.

Let us show that $l_{0}$ is the unique minimizer of the total energy when $t<\mathrm{t}_{\mathrm{i}}$. From (68) and (69), we get for all $l \in\left[l_{0}, L\right]$ and all $t \leq \mathrm{t}_{\mathrm{i}}$ :

$$
\mathscr{E}(t, l)-\mathscr{E}\left(t, l_{0}\right)=-t^{2}\left(\mathscr{P}_{l_{0}}-\mathscr{P}_{l}\right)+\mathrm{G}_{\mathrm{c}}\left(l-l_{0}\right) \geq t^{2} \bar{g}(l) \geq 0 .
$$

Moreover, the inequalities above are equalities if and only if $l=l_{0}$ when $t<\mathrm{t}_{\mathrm{i}}$ and the result follows. Using the same estimates, we can deduce that $l_{0}$ and $l_{\mathrm{i}}$ are the two minimizers of the total energy at $t=\mathrm{t}_{\mathrm{i}}$.

Let us show now that the minimizer is in the open interval $\left(l_{\mathrm{i}}, L\right)$ when $t>\mathrm{t}_{\mathrm{i}}$. From (68) and (69), we get for all $l \in\left[l_{0}, l_{\mathrm{i}}\right.$ ) and all $t>\mathrm{t}_{\mathrm{i}}$ :

$$
\begin{aligned}
\mathscr{E}(t, l)-\mathscr{E}\left(t, l_{\mathrm{i}}\right) & =t^{2}\left(\mathscr{P}_{l}-\mathscr{P}_{l_{\mathrm{i}}}\right)-\mathrm{G}_{\mathrm{c}}\left(l_{\mathrm{i}}-l\right)>\mathrm{t}_{\mathrm{i}}{ }^{2}\left(\mathscr{P}_{l}-\mathscr{P}_{l_{\mathrm{i}}}-\mathrm{G}_{\mathrm{i}}\left(l_{\mathrm{i}}-l\right)\right) \\
& =\mathrm{t}_{\mathrm{i}}{ }^{2}\left(\bar{g}(l)-\bar{g}\left(l_{\mathrm{i}}\right)\right)=\mathrm{t}_{\mathrm{i}}{ }^{2} \bar{g}(l) \geq 0 .
\end{aligned}
$$

Hence, the minimizer cannot be in $\left[l_{0}, l_{\mathrm{i}}\right)$. Since the derivative of the total energy at $l=l_{\mathrm{i}}$ is equal to $\mathrm{G}_{\mathrm{c}}-t^{2} \mathrm{G}_{\mathrm{i}}<0, l_{\mathrm{i}}$ is not the minimizer. In the same manner, since the derivative of the total energy at $l=L$ is equal to $\mathrm{G}_{\mathrm{c}}-t^{2} \varphi_{L}=\mathrm{G}_{\mathrm{c}}>0, L$ cannot be the minimizer. Therefore, the minimizer is in the interval $\left(l_{\mathrm{i}}, L\right)$ when $t>\mathrm{t}_{\mathrm{i}}$. Hence, it must be such that the derivative of the total energy vanishes, which yields $t^{2} \varphi_{l(t)}=\mathrm{G}_{\mathrm{c}}$. Since $l \mapsto \mathscr{G}_{l}$ is monotonically decreasing from $\mathrm{G}_{\mathrm{i}}$ to 0 when $l$ goes 
from $l_{\mathrm{i}}$ to $L$, there exists a unique $l(t) \in\left(l_{\mathrm{i}}, L\right)$ such that $\varphi_{l(t)}=\mathrm{G}_{\mathrm{c}} / t^{2}<\mathrm{G}_{\mathrm{i}}$. The proof of the first part is complete.

4.3. Computation of the crack nucleation by the MAM. Let us consider the cases where $\epsilon$ is sufficiently small in order that $l \mapsto \mathscr{G}_{l}$ be well approximated by the first two nontrivial terms of its matched asymptotic expansion for $l$ in the interval $\left[0,2 l_{\mathrm{m}}\right]$; see $(\mathbf{C 4})$. Accordingly, we have

$$
\frac{\mathscr{G}_{l}}{\mu H} \approx 2 \lambda\left|P_{2}\right|\left(\frac{l}{H}\right)^{2 \lambda-1}-4 \lambda\left|P_{4}\right|\left(\frac{l}{H}\right)^{4 \lambda-1},
$$

using the fact that $P_{2}<0$ and $P_{4}>0$. Therefore, the length $l_{\mathrm{m}}$ where $\varphi_{l}$ is maximal and the maximum $G_{m}$ are approximated by

$$
\frac{l_{\mathrm{m}}}{H} \approx\left(\frac{(2 \lambda-1)\left|P_{2}\right|}{2(4 \lambda-1)\left|P_{4}\right|}\right)^{\frac{1}{2 \lambda}}, \quad \frac{\mathrm{G}_{\mathrm{m}}}{\mu H} \approx \frac{4 \lambda^{2}\left|P_{2}\right|}{4 \lambda-1}\left(\frac{(2 \lambda-1)\left|P_{2}\right|}{2(4 \lambda-1)\left|P_{4}\right|}\right)^{\frac{2 \lambda-1}{2 \lambda}} .
$$

Comparing with the values obtained by the FEM (see (P3) and Table 5), it appears that the agreement is very good for the maximum $\mathrm{G}_{\mathrm{m}}$, and less good for $l_{\mathrm{m}}$. The reason is that the localization of $l_{\mathrm{m}}$ by the FEM is quite imprecise because the graph of $\varphi_{l}$ is very flat near $l_{\mathrm{m}}$ : for instance, for $\epsilon=0.3, \mathscr{G}_{l}$ computed at $\tilde{l}=0.092$ is equal to 0.27327 while it is equal to 0.27307 at $\tilde{l}=0.082$, with a relative difference less than $10^{-4}$.

One can see also in Table 5 that the contribution of the next term, i.e., MAM 6 , is weak when $\epsilon$ is less than 0.2. Its influence, in particular on $l_{\mathrm{i}}$, can no longer be neglected when $\epsilon \geq 0.3$. Note also that MAM 4 underestimates while MAM 6 overestimates the lengths $l_{\mathrm{m}}$ and $l_{\mathrm{i}}$. This bounding property is due to the alternating of the sign of the coefficients $P_{2 i}$ with $i$. However, it is checked numerically only; we are not able to prove it. Using MAM 4 to calculate the nucleation, we obtain the following result:

Proposition 9. In the case of a genuine notch $\epsilon>0$ :

(1) If the body does not contain a preexisting crack $\left(l_{0}=0\right)$, then the time $\mathrm{t}_{\mathrm{i}}$ at which the crack nucleates and the length $l_{\mathrm{i}}$ of the nucleated crack at this time are approximated with the MAM 4 by

$$
\begin{aligned}
& \frac{l_{\mathrm{i}}}{H} \approx 2^{\frac{1}{2 \lambda}} \frac{l_{\mathrm{m}}}{H} \approx\left(\frac{(2 \lambda-1)\left|P_{2}\right|}{(4 \lambda-1)\left|P_{4}\right|}\right)^{\frac{1}{2 \lambda}}, \\
& \mathrm{t}_{\mathrm{i}}{ }^{2} \approx \frac{1}{\lambda 2^{\frac{1}{2 \lambda}}} \frac{\mathrm{G}_{\mathrm{c}}}{\mathrm{G}_{\mathrm{m}}} \approx \frac{\mathrm{t}_{\mathrm{c}}{ }^{2}}{8 \lambda^{3}}\left(\frac{4 \lambda-1}{\left|P_{2}\right|}\right)^{2-\frac{1}{2 \lambda}}\left(\frac{4 P_{4}}{2 \lambda-1}\right)^{1-\frac{1}{2 \lambda}},
\end{aligned}
$$

where $\mathrm{t}_{\mathrm{c}}{ }^{2}=\mathrm{G}_{\mathrm{c}} / \mu H$. 


\begin{tabular}{|c|ccccc|}
\hline$\epsilon$ & 0 & 0.1 & 0.2 & 0.3 & 0.4 \\
$\lambda$ & 0.5 & 0.5164 & 0.5335 & 0.5511 & 0.5689 \\
\hline$l_{\mathrm{m}} / H$ by FEM & 0 & 0.024 & 0.058 & 0.092 & 0.130 \\
$l_{\mathrm{m}} / H$ by MAM 4 & 0 & 0.0255 & 0.0533 & 0.0823 & 0.1124 \\
$l_{\mathrm{m}} / H$ by MAM 6 & 0 & 0.0267 & 0.0584 & 0.0953 & 0.1387 \\
$\mathrm{G}_{\mathrm{m}} / \mu H$ by FEM & 0.4820 & 0.3900 & 0.3260 & 0.2733 & 0.2279 \\
$\mathrm{G}_{\mathrm{m}} / \mu H$ by MAM 4 & 0.4820 & 0.3917 & 0.3264 & 0.2724 & 0.2257 \\
$\mathrm{G}_{\mathrm{m}} / \mu H$ by MAM 6 & 0.4820 & 0.3917 & 0.3274 & 0.2743 & 0.2287 \\
$l_{\mathrm{i}} / H$ by FEM & 0 & 0.0517 & 0.1131 & 0.1814 & 0.2561 \\
$l_{\mathrm{i}} / H$ by MAM 4 & 0 & 0.0499 & 0.1020 & 0.1544 & 0.2067 \\
$l_{\mathrm{i}} / H$ by MAM 6 & 0 & 0.0530 & 0.1163 & 0.1923 & 0.2964 \\
$\mathrm{G}_{\mathrm{i}} / \mu H$ by FEM & 0.4820 & 0.3864 & 0.3195 & 0.2650 & 0.2188 \\
$\mathrm{G}_{\mathrm{i}} / \mu H$ by MAM 4 & 0.4820 & 0.3877 & 0.3195 & 0.2635 & 0.2157 \\
$\mathrm{G}_{\mathrm{i}} / \mu H$ by MAM 6 & 0.4820 & 0.3881 & 0.3208 & 0.2662 & 0.2201 \\
$\mathrm{t}_{\mathrm{i}} / \mathrm{t}_{\mathrm{c}}$ by FEM & 1.440 & 1.605 & 1.766 & 1.938 & 2.132 \\
$\mathrm{t}_{\mathrm{i}} / \mathrm{t}_{\mathrm{c}}$ by MAM 4 & 1.440 & 1.606 & 1.769 & 1.916 & 2.153 \\
$\mathrm{t}_{\mathrm{i}} / \mathrm{t}_{\mathrm{c}}$ by MAM 6 & 1.440 & 1.605 & 1.766 & 1.938 & 2.131 \\
\hline
\end{tabular}

Table 5. Comparisons of the values of $l_{\mathrm{m}}, \mathrm{G}_{\mathrm{m}}, l_{\mathrm{i}}, \mathrm{G}_{\mathrm{i}}$ and $\mathrm{t}_{\mathrm{i}}$ obtained by the FEM with those obtained by MAM 4 and MAM 6 .

(2) If the body contains a preexisting crack of length $l_{0}$ such that $0<l_{0}<l_{\mathrm{m}}$, then the length $l_{\mathrm{i}}$ at which the crack jumps at the onset of the propagation is the unique solution greater than $l_{\mathrm{m}}$ of

$$
\begin{aligned}
0=\left|P_{2}\right|\left((2 \lambda-1) l_{\mathrm{i}}^{2 \lambda}-2 \lambda l_{0} l_{\mathrm{i}}^{2 \lambda-1}\right. & \left.+l_{0}^{2 \lambda}\right) H^{2 \lambda} \\
& -P_{4}\left((4 \lambda-1) l_{\mathrm{i}}^{4 \lambda}+4 \lambda l_{0} l_{\mathrm{i}}^{4 \lambda-1}-l_{0}^{4 \lambda}\right),
\end{aligned}
$$

while the time $\mathrm{t}_{\mathrm{i}}$ at which the onset occurs is given by $\mathrm{t}_{\mathrm{i}}^{2}=\mathrm{G}_{\mathrm{c}} / \mathscr{G}_{l_{\mathrm{i}}}$. Therefore, $l_{\mathrm{i}}$ and $\mathrm{t}_{\mathrm{i}}$ decrease from the values given by (72) to $l_{\mathrm{m}}$ and $\sqrt{\mathrm{G}_{\mathrm{c}} / \mathrm{G}_{\mathrm{m}}}$ given by (71) when $l_{0}$ runs from 0 to $l_{\mathrm{m}}$.

Proof. When $l_{0}=0$, using MAM 4, then (68) becomes

$$
0=(2 \lambda-1)\left|P_{2}\right|\left(\frac{l_{\mathrm{i}}}{H}\right)^{2 \lambda}-(4 \lambda-1)\left|P_{4}\right|\left(\frac{l_{\mathrm{i}}}{H}\right)^{4 \lambda} .
$$

Using (71), (72) can be deduced after some calculations left to the reader. In the same manner, (73) is a direct consequence of (68) and (70). The monotonicity of $l_{\mathrm{i}}$ and $t_{i}$ with respect to $l_{0}$ is easily checked from the graphical interpretation of (73); see Figure 8. 


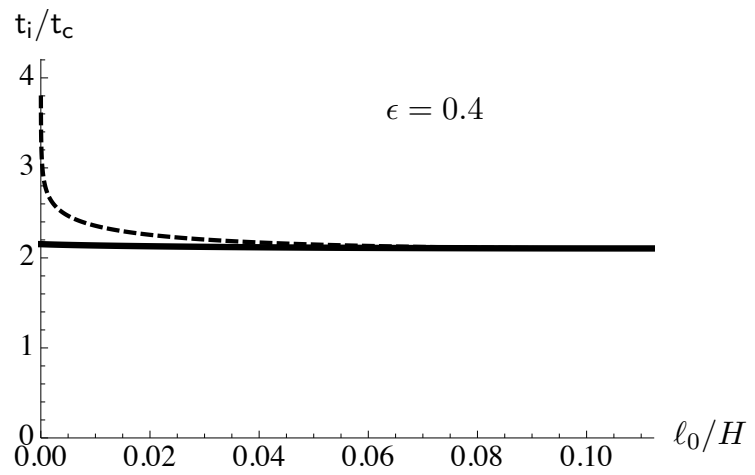

Figure 9. Time at which a preexisting crack starts as a function of its length in the case where the notch parameter $\epsilon$ equals 0.4 . Plain line: from the FM-law; dashed line: from the G-law.

Therefore, since $1 / 2<\lambda<1$ for a genuine notch, the length of the nucleated crack $l_{\mathrm{i}}$ is less than $2 l_{\mathrm{m}}$ while the critical time $\mathrm{t}_{\mathrm{i}}$ is not greater than $2^{1 / 4} \sqrt{\mathrm{G}_{\mathrm{c}} / \mathrm{G}_{\mathrm{m}}}$. For a very sharp notch, i.e., when $\epsilon$ is small, then $2 \lambda \approx 1+\epsilon / \pi$ and

$$
l_{\mathrm{i}} \approx \frac{\epsilon\left|P_{2}\right|}{\pi P_{4}} H, \quad \mathrm{t}_{\mathrm{i}}^{2} \approx \frac{\mathrm{G}_{\mathrm{c}}}{\left|P_{2}\right| \mu H},
$$

where $P_{2} \approx-0.4820$ and $P_{4} \approx 0.3282$. Therefore we recover the response associated with a crack when the notch angle tends to $2 \pi$. The FM-law delivers an evolution which depends continuously on the parameter $\epsilon$, in contrast with the G-law.

As long as the dependence of $t_{i}$ on $l_{0}$ is concerned, it turns out that the FM-law predicts that the variation of $\mathrm{t}_{\mathrm{i}}$ is small when $l_{0}$ goes from 0 to $l_{\mathrm{m}}$ as can be seen in Figure 9 for $\epsilon=0.4$. Indeed, $\mathrm{t}_{\mathrm{i}} / \mathrm{t}_{\mathrm{c}}$ decreases from 2.153 to 2.105 when $l_{0}$ varies from 0 to $l_{\mathrm{m}}=0.112 \mathrm{H}$. This constitutes also a strong difference with the prediction of the $G$-law for which $t_{i}$ goes to infinity when $l_{0}$ goes to 0 .

\section{Conclusion and perspectives}

We have presented here a general method based on matched asymptotic expansions which can be applied to determine the mechanical fields and all related mechanical quantities in the case of a defect located at the tip of a notch. Applying this method to the case of a noncohesive crack, it turns out that it is sufficient to solve a few inner and outer problems to compute with very good accuracy the dependency of the energy and the energy release rate on the length of the crack. Moreover, this approximation can be used for very small values of the length of the crack and hence to determine the onset of the cracking, whereas a classical finite element 
method gives rise to inaccurate results. In particular, the matched asymptotic method permits a comparison of the nucleation process of a crack at the tip of the notch which is predicted by the classical Griffith criterion with that predicted by the principle of energy minimization proposed in [Francfort and Marigo 1998]. It turns out that the latter principle gives rise to much more relevant results than the former, from a physical viewpoint.

A natural extension of this work is to consider situations where the geometry and the loading have no symmetry and hence the direction that the nucleated crack will choose must also be predicted. Let us note that the G-law alone is not able to give an answer, and another criterion must be supplemented. In an antiplane setting, the principle of local symmetry, which is by essence made for an isotropic plane setting, cannot be used. It turns out that the FM-law in its general statement can also predict the direction and more generally the path of the crack; see [Chambolle et al. 2009; 2010; Francfort and Marigo 1998]. So, an interesting challenge should be to use the MAM and the FM-law in a nonsymmetric case to predict also the direction of nucleation. Another natural and desirable extension of the present work is to develop the method in a plane elasticity setting. It seems that there is no conceptual difficulty in doing that. The last perspective concerns the choice of the surface energy. Indeed, the present study is based on the crucial Griffith assumption that the surface energy is proportional to the crack area. This assumption has very important consequences on the nucleation as we have seen in the paper. With this hypothesis, there is no cohesive force and hence the model does not contain the concept of critical stress. An important step will be to apply the MAM in the case of a cohesive crack [Barenblatt 1962; Dugdale 1960; Del Piero and Raous 2010], which automatically contains a critical stress and even a characteristic length. The goal will be to study the influence of the critical stress and characteristic length on the nucleation and the propagation of a crack in the spirit of the previous works based on the variational approach to fracture [Abdelmoula et al. 2010; Bourdin et al. 2008; Charlotte et al. 2006; Del Piero and Truskinovsky 2009; Ferdjani et al. 2007; Giacomini 2005; Jaubert and Marigo 2006; Marigo and Truskinovsky 2004].

\section{References}

[Abdelmoula and Marigo 2000] R. Abdelmoula and J.-J. Marigo, "The effective behavior of a fiber bridged crack", J. Mech. Phys. Solids 48:11 (2000), 2419-2444.

[Abdelmoula et al. 2010] R. Abdelmoula, J.-J. Marigo, and T. Weller, "Construction and justification of Paris-like fatigue laws from Dugdale-type cohesive models", Ann. Solid Struct. Mech. 1:3-4 (2010), 139-158.

[Barenblatt 1962] G. I. Barenblatt, "The mathematical theory of equilibrium cracks in brittle fracture", pp. 55-129 in Advances in Applied Mechanics, vol. 7, Academic Press, New York, 1962.

[Bilteryst and Marigo 2003] F. Bilteryst and J.-J. Marigo, "An energy based analysis of the pull-out problem", Eur. J. Mech. A Solids 22:1 (2003), 55-69. 
[Bonnaillie-Noël et al. 2010] V. Bonnaillie-Noël, M. Dambrine, F. Hérau, and G. Vial, "On generalized Ventcel's type boundary conditions for Laplace operator in a bounded domain", SIAM J. Math. Anal. 42:2 (2010), 931-945.

[Bonnaillie-Noel et al. 2011] V. Bonnaillie-Noel, M. Dambrine, and G. Vial, "Small defects in mechanics", AIP Conference Proceedings 1389:1 (2011), 1416-1419.

[Bourdin et al. 2008] B. Bourdin, G. A. Francfort, and J.-J. Marigo, "The variational approach to fracture", J. Elasticity 91:1-3 (2008), 5-148.

[Bui 1978] H. Bui, Mécanique de la rupture fragile, Masson, 1978.

[Chambolle et al. 2008] A. Chambolle, A. Giacomini, and M. Ponsiglione, "Crack initiation in brittle materials", Arch. Ration. Mech. Anal. 188:2 (2008), 309-349.

[Chambolle et al. 2009] A. Chambolle, G. A. Francfort, and J.-J. Marigo, "When and how do cracks propagate?", J. Mech. Phys. Solids 57:9 (2009), 1614-1622.

[Chambolle et al. 2010] A. Chambolle, G. A. Francfort, and J.-J. Marigo, "Revisiting energy release rates in brittle fracture", J. Nonlinear Sci. 20:4 (2010), 395-424.

[Charlotte et al. 2006] M. Charlotte, J. Laverne, and J.-J. Marigo, "Initiation of cracks with cohesive force models: a variational approach”, Eur. J. Mech. A Solids 25:4 (2006), 649-669.

[Cherepanov 1979] G. Cherepanov, Mechanics of brittle fracture, McGraw-Hill, New York, 1979.

[Dauge 1988] M. Dauge, Elliptic boundary value problems on corner domains: Smoothness and asymptotics of solutions, Lecture Notes in Mathematics 1341, Springer, Berlin, 1988.

[Dauge et al. 2010] M. Dauge, S. Tordeux, and G. Vial, "Selfsimilar perturbation near a corner: matching versus multiscale expansions for a model problem", pp. 95-134 in Around the research of Vladimir Maz'ya, II, edited by A. Laptev, Int. Math. Ser. (N. Y.) 12, Springer, New York, 2010.

[David et al. 2012] M. David, J.-J. Marigo, and C. Pideri, "Homogenized interface model describing inhomogeneities located on a surface”, J. Elasticity 109:2 (2012), 153-187.

[Del Piero and Raous 2010] G. Del Piero and M. Raous, "A unified model for adhesive interfaces with damage, viscosity, and friction”, Eur. J. Mech. A Solids 29:4 (2010), 496-507.

[Del Piero and Truskinovsky 2009] G. Del Piero and L. Truskinovsky, "Elastic bars with cohesive energy", Contin. Mech. Thermodyn. 21:2 (2009), 141-171.

[Destuynder and Djaoua 1981] P. Destuynder and M. Djaoua, "Sur une interprétation mathématique de l'intégrale de Rice en théorie de la rupture fragile", Math. Methods Appl. Sci. 3:1 (1981), 70-87.

[Dugdale 1960] D. Dugdale, "Yielding of steel sheets containing slits", J. Mech. Phys. Solids 8:2 (1960), 100-104.

[Ferdjani et al. 2007] H. Ferdjani, R. Abdelmoula, and J.-J. Marigo, "Insensitivity to small defects of the rupture of materials governed by the Dugdale model", Contin. Mech. Thermodyn. 19:3-4 (2007), 191-210.

[Francfort and Marigo 1998] G. A. Francfort and J.-J. Marigo, "Revisiting brittle fracture as an energy minimization problem”, J. Mech. Phys. Solids 46:8 (1998), 1319-1342.

[Geymonat et al. 2011] G. Geymonat, F. Krasucki, S. Hendili, and M. Vidrascu, "The matched asymptotic expansion for the computation of the effective behavior of an elastic structure with a thin layer of holes", Int. J. Multiscale Comput. Eng. 9:5 (2011), 529-542.

[Giacomini 2005] A. Giacomini, "Size effects on quasi-static growth of cracks", SIAM J. Math. Anal. 36:6 (2005), 1887-1928.

[Griffith 1921] A. A. Griffith, "The phenomena of rupture and flow in solids", Phil. Trans. R. Soc. Lond. A 221 (1921), 163-198. 
[Grisvard 1985] P. Grisvard, Elliptic problems in nonsmooth domains, Monographs and Studies in Mathematics 24, Pitman (Advanced Publishing Program), Boston, 1985.

[Grisvard 1986] P. Grisvard, "Problèmes aux limites dans les polygones: Mode d'emploi", EDF Bull. Direction Études Rech. Sér. C Math. Inform. 1 (1986), 21-59.

[Irwin 1958] G. R. Irwin, "Fracture", pp. 551-590 in Handbuch der Physik, Band 6: Elastizität und Plastizität, edited by S. Flügge, Springer, Berlin, 1958.

[Jaubert and Marigo 2006] A. Jaubert and J.-J. Marigo, "Justification of Paris-type fatigue laws from cohesive forces model via a variational approach", Contin. Mech. Thermodyn. 18:1-2 (2006), 23-45.

[Lawn 1993] B. Lawn, Fracture of brittle solids, 2nd ed., Cambridge University Press, 1993.

[Leblond 2003] J.-B. Leblond, Mécanique de la rupture fragile et ductile, Hermes Science Publications, Paris, 2003.

[Leguillon 1989] D. Leguillon, “Calcul du taux de restitution de l'énergie au voisinage d'une singularité”, C. R. Acad. Sci., Paris, Sér. II 309:10 (1989), 945-950.

[Marigo 2010] J.-J. Marigo, "Initiation of cracks in Griffith's theory: An argument of continuity in favor of global minimization”, J. Nonlinear Sci. 20:6 (2010), 831-868.

[Marigo and Pideri 2011] J.-J. Marigo and C. Pideri, "The effective behavior of elastic bodies containing microcracks or microholes localized on a surface", Int. J. Damage Mech. 20:8 (2011), 11511177.

[Marigo and Truskinovsky 2004] J.-J. Marigo and L. Truskinovsky, "Initiation and propagation of fracture in the models of Griffith and Barenblatt", Contin. Mech. Thermodyn. 16:4 (2004), 391-409.

[Negri 2010] M. Negri, "A comparative analysis on variational models for quasi-static brittle crack propagation”, Adv. Calc. Var. 3:2 (2010), 149-212.

[Negri and Ortner 2008] M. Negri and C. Ortner, "Quasi-static crack propagation by Griffith's criterion”, Math. Models Methods Appl. Sci. 18:11 (2008), 1895-1925.

[Nguyen 2000] Q. S. Nguyen, Stability and nonlinear solid mechanics, Wiley, Chichester, 2000.

[Rice 1968] J. R. Rice, "A path independent integral and the approximate analysis of strain concentration by notches and cracks", J. Appl. Mech. 35:2 (1968), 379-386.

[Vidrascu et al. 2012] M. Vidrascu, G. Geymonat, S. Hendili, and F. Krasucki, "Matched asymptotic expansion and domain decomposition for an elastic structure", pp. 281 in Twenty-first international conference on domain decomposition methods (Rennes, France, June 25-29, 2012), 2012.

Received 18 Dec 2012. Revised 30 Apr 2013. Accepted 5 Jun 2013.

THI BACH TUYET DANG: tuyet@lms.polytechnique.fr

Laboratoire de Mécanique des Solides, École Polytechnique, CNRS, UMR 7649,

91128 Palaiseau cedex, France

LAURENCE HALPERN: halpern@math .univ-paris13.fr

LAGA, Université Paris 13, Sorbonne Paris Cité, CNRS, UMR 7539, 93430 Villetaneuse, France

JEAN-JACQUES MARIGO: marigo@lms .polytechnique.fr

Laboratoire de Mécanique des Solides, École Polytechnique, CNRS, UMR 7649,

91128 Palaiseau cedex, France 


\section{EDITORIAL BOARD}

ANTONIO CARCATERRA ERIC A. CARLEN

FRANCESCO DELL'ISOLA

RAFFAELE ESPOSITO

ALBERT FANNJIANG

Gilles A. FrancFort

PiERANGElo MARCATI

JEAN-JACQUES MARIGO

PETER A. MARKOWICH MARTIN OSTOJA-STARZEWSKI

PIERRE SEPPECHER

DAVID J. STEIGMANN

PAUl STEINMANN

PierRe M. Suquet

\section{MANAGING EDITORS}

MICOL AMAR

CORRADO LATTANZIO

ANGELA MADEO

MARTIN OSTOJA-STARZEWSKI

\section{ADVISORY BOARD}

ADNAN AKAY

Holm AltenbaCH

MICOL AMAR

HARM ASKES

TEODOR ATANACKOVIĆ

VICTOR BERDICHEVSKY

GuY BouchitTÉ

ANDREA BRAIDES

ROBERTO CAMASSA

MAURO CARFORE

ERIC DARVE

FELIX DARVE

ANNA DE MASI

Gianpietro Del Piero

EMMANUELE Di BENEDETTO

BERNOLD FIEDLER

IRENE M. GAMBA

SERGEY GAVRILYUK

TIMOTHY J. HEALEY

DOMINIQUE JEULIN

ROGER E. KHAYAT

CORRADO LATTANZIO

ROBERT P. LIPTON

ANGELO LUONGO

ANGEla MadeO

JUAN J. MANFREDI

CARLO MARCHIORO

GÉrard A. MAUGin

ROBERTO NATALINI

PATRIZIO NEFF

ANDREY PIATNITSKI

ERrico Presutti

MARIO PUlVIRENTI

LuCiO RuSSO

Miguel A. F. SANJUAN

Patrick SElvadurai

ALEXANDER P. SEYRANIAN

MIROSLAV ŠILHAVÝ

GUIDO SWEERS

ANTOINETTE TORDESILLAS

LEV TRUSKINOVSKY

JUAN J. L. VELÁZQUEZ

VINCENZO VESPRI

ANGELO VULPIANI msp.org/memocs

Università di Roma “La Sapienza”, Italia

Rutgers University, USA

(CO-CHAIR) Università di Roma "La Sapienza", Italia

(TREASURER) Università dell'Aquila, Italia

University of California at Davis, USA

(CO-CHAIR) Université Paris-Nord, France

Università dell' Aquila, Italy

École Polytechnique, France

DAMTP Cambridge, UK, and University of Vienna, Austria

(CHAIR MANAGING EDITOR) Univ. of Illinois at Urbana-Champaign, USA

Université du Sud Toulon-Var, France

University of California at Berkeley, USA

Universität Erlangen-Nürnberg, Germany

LMA CNRS Marseille, France

Università di Roma “La Sapienza”, Italia

Università dell' Aquila, Italy

Université de Lyon-INSA (Institut National des Sciences Appliquées), France (CHAIR MANAGING EDITOR) Univ. of Illinois at Urbana-Champaign, USA

Carnegie Mellon University, USA, and Bilkent University, Turkey

Otto-von-Guericke-Universität Magdeburg, Germany

Università di Roma "La Sapienza”, Italia

University of Sheffield, UK

University of Novi Sad, Serbia

Wayne State University, USA

Université du Sud Toulon-Var, France

Università di Roma Tor Vergata, Italia

University of North Carolina at Chapel Hill, USA

Università di Pavia, Italia

Stanford University, USA

Institut Polytechnique de Grenoble, France

Università dell'Aquila, Italia

Università di Ferrara and International Research Center MEMOCS, Italia

Vanderbilt University, USA

Freie Universität Berlin, Germany

University of Texas at Austin, USA

Université Aix-Marseille, France

Cornell University, USA

École des Mines, France

University of Western Ontario, Canada

Università dell' Aquila, Italy

Louisiana State University, USA

Università dell' Aquila, Italia

Université de Lyon-INSA (Institut National des Sciences Appliquées), France

University of Pittsburgh, USA

Università di Roma "La Sapienza", Italia

Université Paris VI, France

Istituto per le Applicazioni del Calcolo "M. Picone", Italy

Universität Duisburg-Essen, Germany

Narvik University College, Norway, Russia

Università di Roma Tor Vergata, Italy

Università di Roma "La Sapienza", Italia

Università di Roma “Tor Vergata", Italia

Universidad Rey Juan Carlos, Madrid, Spain

McGill University, Canada

Moscow State Lomonosov University, Russia

Academy of Sciences of the Czech Republic

Universität zu Köln, Germany

University of Melbourne, Australia

École Polytechnique, France

Bonn University, Germany

Università di Firenze, Italia

Università di Roma La Sapienza, Italia

MEMOCS (ISSN 2325-3444 electronic, 2326-7186 printed) is a journal of the International Research Center for the Mathematics and Mechanics of Complex Systems at the Università dell'Aquila, Italy.

Cover image: "Tangle" by @ John Horigan; produced using the Context Free program (contextfreeart.org).

\section{PUBLISHED BY}

mathematical sciences publishers

nonprofit scientific publishing

http://msp.org/

(C) 2014 Mathematical Sciences Publishers 
Mathematics and Mechanics of Complex Systems vol. 2 no. 2

A mixed boundary value problem in potential theory for a bimaterial porous region: An application in the environmental geosciences

A. P. S. Selvadurai

Geometric degree of nonconservativity

Jean Lerbet, Marwa Aldowaji, Noël Challamel, Oleg N.

Kirillov, François Nicot and Félix Darve

Asymptotic analysis of small defects near a singular point in antiplane elasticity, with an application to the nucleation of a crack at a notch

Thi Bach Tuyet Dang, Laurence Halpern and Jean-Jacques Marigo

The homogenized behavior of unidirectional fiber-reinforced composite materials in the case of debonded fibers

Yahya Berrehili and Jean-Jacques Marigo

Statistically isotropic tensor random fields: Correlation structures

Anatoliy Malyarenko and Martin Ostoja-Starzewski

MEMOCS is a journal of the International Research Center for the Mathematics and Mechanics of Complex Systems at the Università dell' Aquila, Italy.

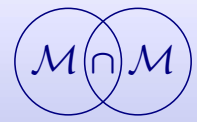

